\title{
Domain dependence of eigenvalues of elliptic type operators
}

\author{
Vladimir Kozlov (Linköping)* \\ May 23, 2019 \\ Department of Mathematics, Linköping University, S-581 83 Linköping, \\ Sweden \\ E-mail address: vlkoz@mai.liu.se
}

\begin{abstract}
The dependence on the domain is studied for the Dirichlet eigenvalues of an elliptic operator considered in bounded domains. Their proximity is measured by a norm of the difference of two orthogonal projectors corresponding to the reference domain and the perturbed one; this allows to compare domains that have non-smooth boundaries and different topology. The main result is an asymptotic formula in which the remainder is evaluated in terms of this quantity. As an application, the stability of eigenvalues is estimated by virtue of integrals of squares of the gradients of eigenfunctions for elliptic problems in different domains. It occurs that these stability estimates imply well-known inequalities for perturbed eigenvalues.
\end{abstract}

\section{Introduction}

We consider eigenvalues of the Dirichlet problem for an elliptic operator in a domain in $\mathbb{R}^{n}, n \geq 2$. Our main aim is to study how these eigenvalues depend on the domain. The first results concerning this classical problem can be found presumably in the book [16] by Rayleigh. A general technique was proposed by Hadamard [8], [9], who studied perturbations of a domain with a smooth boundary. In his works, the boundary of the perturbed domain

*The author was supported by the Swedish Research Council (VR) 
$\Omega_{\varepsilon}$ is described by the function $\tau=\varepsilon h\left(x^{\prime}\right)$, where $\tau$ is the variable along the normal to the boundary, $h$ is a smooth function on the boundary and $\varepsilon$ is a small parameter. Hadamard's formula for the perturbed first eigenvalue $\lambda\left(\Omega_{\varepsilon}\right)$ of the Dirichlet Laplacian is as follows:

$$
\lambda\left(\Omega_{\varepsilon}\right)=\lambda\left(\Omega_{0}\right)+\varepsilon \int_{\partial \Omega_{0}}\left|\partial_{\tau} \varphi\right|^{2} h d S+o(\varepsilon),
$$

where $\varphi$ is the first eigenfunction such that $\|\varphi\|_{L^{2}}=1$ and $d S$ is the surface measure on $\partial \Omega_{0}$. In various generalizations of this formula, perturbations are described by a family of smooth mappings, other boundary conditions are considered as well as more general elliptic operators; see [4, [1], 3], 15], [10] and references cited therein. Some non-smooth perturbations of smooth boundaries, that are described by normal shift functions, were treated in [5]-[7], [17]. On the other hand, there are many problems involving more general classes of perturbations, namely, non-smooth perturbations of nonsmooth boundaries and perturbations that cannot be described by a family of isomorphisms.

In [12]-[14], another approach was proposed to studying the dependence of $\lambda$ on the domain and the operator's coefficients. It is based on an abstract theorem concerning perturbation of eigenvalues for operators acting in different spaces. Here we further develop the approach used in [12]. In this paper, the main novelty is the application of a new small parameter to measure the proximity of two spectral problems. This parameter is a norm of the difference of orthogonal projectors on Sobolev spaces consisting of functions given on these domains. In Sect. 2.1, we show that the convergence with respect to this parameter (it is actually a distance) is equivalent to the convergence in the sense of Mosco or to $\gamma$-convergence, see [10] and [1]. The latter type of convergence plays an important role in proving the existence of solutions to various shape optimization problems dealing with eigenvalues. Another new point is a refined estimate of the remainder term. It allows us to extend essentially the class of domains and their perturbations for which a certain "generalized" asymptotic formula is still valid. Let us turn to a detailed description of the results. For this purpose we shall use an example of second order elliptic operator.

Let $\Omega_{1}$ and $\Omega_{2}$ be two bounded domains with nonempty intersection. We 
consider a bilinear form

$$
(u, v)=\sum_{i, j=1}^{n} \int_{\mathbb{R}^{n}} A_{i j}(x) \partial_{x_{j}} u \partial_{x_{i}} \bar{v} d x,
$$

where $A_{i j}$ are bounded measurable real-valued functions such that $A_{i j}=A_{j i}$ and

$$
\nu|\xi|^{2} \leq \sum_{i, j=1}^{n} A_{i j}(x) \xi_{i} \xi_{j} \leq \nu^{-1}|\xi|^{2}
$$

for all $\xi \in \mathbb{R}^{n} \backslash \mathcal{O}$ and $x \in \mathbb{R}^{n}$. The form $(\cdot, \cdot)$ defines a new inner product in the space $H_{k}=\stackrel{\circ}{W}^{1,2}\left(\Omega_{k}\right), k=1,2$ (we suppose that functions belonging to both of these spaces are extended by zero to the whole $\mathbb{R}^{n}$ ); the corresponding norm will be denoted by $\|\cdot\|$. Let us consider the following spectral problems

$$
(\varphi, v)=\lambda\langle\varphi, v\rangle \quad \text { for all } v \in H_{1}
$$

and

$$
(U, V)=\mu\langle U, V\rangle \quad \text { for all } V \in H_{2},
$$

where $\langle\cdot, \cdot\rangle$ is the inner product in $L^{2}, \varphi \in H_{1}$ and $U \in H_{2}$. By $\lambda_{m}$ we denote the $m$ th eigenvalue of problem (44); let $X_{m}$ be the corresponding eigenspace, $J_{m}=\operatorname{dim} X_{m}$. Our aim is to describe eigenvalues of problem (5) located near the eigenvalue $\lambda_{m}$ of (4).

If $\Omega_{1}$ and $\Omega_{2}$ are sub-domains of a bounded domain $D$, then by $S_{j}, j=$ 1,2 , we denote the orthogonal projector defined on $\stackrel{\circ}{W}^{1,2}(D)$ whose image belongs to $H_{j}$. For characterizing the proximity of $\Omega_{1}$ and $\Omega_{2}$ we use the best constant $\sigma=\sigma\left(H_{1}, H_{2}\right)$ in the inequality

$$
\left|\left(S_{1}-S_{2}\right) u\right|^{2} \leq \sigma\|u\|^{2}, \quad u \in \stackrel{\circ}{W}^{1,2}(D),
$$

where $|\cdot|$ is the $L^{2}$-norm. In Sect. 2.1, we show that the $\gamma$-convergence of domains is equivalent to their convergence in terms of the distance (6).

If $\sigma$ is sufficiently small, then problem (5) has exactly $J_{m}$ eigenvalues, say $\mu_{1}, \ldots, \mu_{J_{m}}$, in a neighborhood of $\lambda_{m}$; see Proposition 3 , In order to formulate one of our main results we introduce the following notation: $T_{2} u=u-S_{2} u, \Psi=\Psi_{\varphi} \in H_{2}$ is the solution of the equation

$$
(\Psi, w)=(\varphi, w)-\lambda_{m}\langle\varphi, w\rangle \text { for all } w \in H_{2}
$$


and

$$
\left.\rho=\max _{\varphi \in X_{m},\|\varphi\|=1}\left(|T \varphi|^{2}+\left|\Psi_{\varphi}\right|^{2}+\sigma\left\|\Psi_{\varphi}\right\|^{2}\right)\right) .
$$

Now we are in a position to formulate the following.

Theorem 1. The asymptotic formula holds:

$$
\mu_{k}^{-1}=\lambda_{m}^{-1}+\tau_{k}+O\left(\rho+\left|\tau_{k}\right| \sigma\right), \quad k=1, \ldots, J_{m}
$$

Here $\tau=\tau_{k}$ is an eigenvalue of the problem

$\frac{1}{\lambda_{m}}\left(\left(\Psi_{\varphi}, \Psi_{\psi}\right)-(T \varphi, T \psi)-\left(\Psi_{\varphi}, \psi\right)-\left(\varphi, \Psi_{\psi}\right)\right)=\tau(S \varphi, S \psi) \quad$ for all $\psi \in X_{m}$

where $\varphi \in X_{m}$; moreover, $\tau_{1}, \ldots, \tau_{m}$ in (9) run through all eigenvalues of (10) counted according to their multiplicity.

In the case when $\Omega_{2} \subset \Omega_{1}$, the function $\Psi_{\varphi}$ vanishes and the eigenvalue problem (10) takes the form:

$$
-\frac{1}{\lambda_{m}}(T \varphi, T \psi)=\tau(S \varphi, S \psi) \text { for all } \psi \in X_{m}
$$

If $\Omega_{1} \subset \Omega_{2}$, then $T \varphi=0$ and (10) can be written as follows:

$$
\frac{1}{\lambda_{m}}\left(\Psi_{\varphi}, \Psi_{\psi}\right)=\tau(S \varphi, S \psi) \text { for all } \psi \in X_{m}
$$

Similar theorems were proved in [12]1 and [14], but here, the main novelty is the use of the small parameter $\sigma$ which makes the present theorem applicable to a substantially larger class of perturbations. The importance of this parameter, that serves as a distance in the set of all closed subspaces of $W^{1,2}(D)$, lies in the fact that this set is compact with respect to this distance (see Proposition 2). As a result one obtain solutions of various optimization problems for functionals that are continuous with respect to this distance. Another new point is that the remainder in (10) has the form, in which the small parameter $\sigma$ appears explicitly together with the quantities involved in the finite dimensional spectral problem (10).

\footnotetext{
${ }^{1}$ Two terms are lost in formula (5), 12, compare with (9) and (10). However, if $\Omega_{2} \subset \Omega_{1}$ or $\Omega_{1} \subset \Omega_{1}$, then formula (5), [12], is true.
} 
Let us describe some applications of the asymptotic formula (10). We begin with the case when both $\Omega_{1}$ and $\Omega_{2}$ are Lipschitz domains with the Lipschitz constant less than or equal to $C_{*}$ or, what is the same, uniformly Lipschitz (see [11] and [10], where the definitions of these notions are given). We assume that

$$
\Omega_{\varepsilon}=\left\{x \in \Omega_{1}, \operatorname{dist}\left(x, \partial \Omega_{1}\right)>\varepsilon\right\} \subset \Omega_{2} \subset \Omega_{\varepsilon}^{+}=\left\{x \in \mathbb{R}^{n}, \operatorname{dist}\left(x, \Omega_{1}\right)<\varepsilon\right\},
$$

where $\varepsilon$ is a small positive parameter. It is supposed that the constant $C_{*}$ is independent of $\varepsilon$. Then there exists a set $S$, subject to the conditions

$$
\Omega_{1} \backslash \Omega_{2 \varepsilon} \subset S \subset \Omega_{1} \text { and }|S| \leq C_{1}\left|\Omega_{2} \backslash \Omega_{1}\right|,
$$

and such that the following inequality holds:

$$
\left|\mu_{k}^{-1}-\lambda_{m}^{-1}\right| \leq C_{1} \max _{\varphi \in X_{m},\|\varphi\|=1} \int_{\left(\Omega_{1} \backslash \Omega_{2}\right) \cup S}|\nabla \varphi|^{2} d x, k=1, \ldots, J_{m} .
$$

Here $|S|$ is the surface measure of $S$ and the constants $C_{1}$ and $C_{2}$ do not depend on $\varepsilon$. If $\Omega_{2} \subset \Omega_{1}$, then one has that

$$
\left|\mu_{k}^{-1}-\lambda_{m}^{-1}\right| \geq c \min _{\varphi \in X_{m},\|\varphi\|=1} \int_{\left(\Omega_{1} \backslash \Omega_{2}\right)}|\nabla \varphi|^{2} d x, \quad k=1, \ldots, J_{m},
$$

where the integral is the same as in the right-hand side of (14). If we assume that the eigenfunctions $\varphi_{k}, k=1, \ldots, J_{m}$, belong to $W^{1, p}\left(\Omega_{1}\right)$ for some $p \in$ $(2, \infty]$ then formula (14) implies that

$$
\left|\mu_{k}^{-1}-\lambda_{m}^{-1}\right| \leq C\left|\Omega_{1} \triangle \Omega_{2}\right|^{1-\frac{2}{p}},
$$

where $\Omega_{1} \triangle \Omega_{2}$ denotes the symmetric difference of $\Omega_{1}$ and $\Omega_{2}$. Estimate (15) is equivalent to (1.8), [2, but the assumptions imposed on $\Omega_{1}$ and $\Omega_{2}$ are weaker here; in particular, all egenfunctions are required to belong to $W^{1, p}\left(\Omega_{1}\right)$ in $[2$.

Let $\Omega_{1}$ be a bounded domain, $x_{0} \in \partial \Omega_{1}$ and $\varepsilon>0$. We assume that

$$
\Omega_{1} \backslash B_{\varepsilon}\left(x_{0}\right) \subset \Omega_{2} \subset \Omega_{1} \cup B_{\varepsilon}\left(x_{0}\right)=\Omega_{\varepsilon}^{+}\left(x_{0}\right),
$$

where $B_{\varepsilon}\left(x_{0}\right)$ is the ball of the radius $\varepsilon$ centered at $x_{0}$. Let also for all $u \in W^{1,2}\left(B_{q \varepsilon\left(x_{0}\right)}\right)$ such that $u=0$ on $B_{q \varepsilon}\left(x_{0}\right) \backslash \Omega_{\varepsilon}^{+}\left(x_{0}\right)$ the following inequality

$$
\int_{\Omega_{\varepsilon}^{+}\left(x_{0}\right) \cap B_{q \varepsilon\left(x_{0}\right)}}|u|^{2} d x \leq C \varepsilon^{2} \int_{\Omega_{\varepsilon}^{+}\left(x_{0}\right) \cap B_{q \varepsilon}\left(x_{0}\right)}|\nabla u|^{2} d x
$$


hold with some $q>1$ and a constant $C$ independent of $\varepsilon$. Then it is proved in Sect 5.1 that

$$
\left|\mu_{k}^{-1}-\lambda_{m}^{-1}\right| \leq C \max _{\varphi \in X_{m},\|\varphi\|=1} \int_{\Omega_{1} \backslash B_{q \varepsilon}\left(x_{0}\right)}|\nabla \varphi|^{2} d x, k=1, \ldots, J_{m} .
$$

In Sect 5.2, we consider perturbations satisfying (13). Assuming that for some $q>1$ inequality (17) holds for all $x_{0} \in \partial \Omega_{1}$, we prove the following estimate for the perturbed eigenvalues:

$$
\left|\mu_{k}^{-1}-\lambda_{m}^{-1}\right| \leq C \max _{\varphi \in X_{m},\|\varphi\|=1} \int_{\Omega_{1} \backslash \Omega_{q \varepsilon}}|\nabla \varphi|^{2} d x, \quad k=1, \ldots, J_{m} .
$$

Notice that the Sobolev space $\stackrel{\circ}{W}^{1,2}\left(\Omega_{k}\right)$ can be considered as a subspace of a similar Sobolev space in a larger domain. Thus, we present an abstract approach for comparison of eigenvalues and eigenfunctions of operators acting in different subspaces of a certain Hilbert space in Sections 24. There, in order to measure the proximity of two subspaces, we introduce a distance $\sigma$ as a norm of two projectors onto these subspaces and show that the convergence with respect to this distance is equivalent to the Mosco convergence. In Sect. 2, we formulate a proposition about the closeness of eigenvalues and eigenfunctions of two eigenvalue problems, see Proposition 3. In the same section, we present the main asymptotic theorem, see Theorem 2 . Proposition 3 and Theorem 2 are proved in in Sections 3 and 4 , respectively. In Sect. 5. we apply our asymptotic formula to eigenvalues of the Dirichlet problem for a second-order differential operator. In particular, we consider local and global perturbations of the boundary; the case of uniformly Lipschitz boundaries is also treated. A class of domain perturbations, for which the quantity $\sigma$ is small, is described for both cases; a stability estimate, which evaluates eigenvalues by integrals of squares of the gradient of eigenfunctions, is also presented.

\section{Perturbation of eigenvalues. Abstract ver- sion.}

\subsection{Statement of the perturbation problem}

Here we present an abstract approach for study of perturbation of eigenvalues to the spectral problems from Introduction keeping the same notations. 
Let $H$ and $\mathcal{H}$ be Hilbert spaces with inner products $(\cdot, \cdot)$ and $\langle\cdot, \cdot\rangle$ and with corresponding norms $\|\cdot\|$ and $|\cdot|$ respectively. We suppose that $H$ is compactly imbedded in $\mathcal{H}$. This implies existence of $c_{0}>0$ such that

$$
|u| \leq c_{0}|| u \|, \quad \text { for } u \in H .
$$

Let $H_{1}$ and $H_{2}$ be two subspaces of $H$ of infinite dimension. We introduce the operators $K_{j}: H_{j} \rightarrow H_{j}$ by $\left(K_{j} u, v\right)=\langle u, v\rangle$, where $u, v \in H_{j}, j=1,2$. One can check that the operators $K_{1}$ and $K_{2}$ are self-adjoint, positive definite and compact. Let $\mathcal{H}_{j}, j=1,2$, be the closure of $H_{j}$ in the space $\mathcal{H}$. From the definition of $K_{j}, j=1,2$, it follows that the operator can be extended to $\mathcal{H}_{j}$ and

$$
\left\|K_{j}\right\|_{\mathcal{H}_{J} \rightarrow H_{j}} \leq c_{0},
$$

where $c_{0}$ is the constant in (18).

We consider two spectral problems

$$
K_{1} \varphi=\lambda^{-1} \varphi, \varphi \in H_{1},
$$

and

$$
K_{2} U=\mu^{-1} U, \quad U \in H_{2},
$$

We denote by $\lambda_{j}^{-1}, j=1, \ldots$, eigenvalues of $K_{1}$, numerated according to $0<$ $\lambda_{1}<\lambda_{2}<\cdots$, and by $X_{j}$ corresponding eigenspaces. We put $J_{j}=\operatorname{dim} X_{j}$. Our goal is to study eigenvalues of (20) located in a neighborhood of $\lambda_{m}$ for certain fixed $m 2$

We denote by $S_{j}, j=1,2$, the orthogonal projector in $H$ with the image $H_{j}$. We will measure the proximity between $H_{1}$ and $H_{2}$ by the constant $\sigma=\sigma\left(H_{1}, H_{2}\right)$ in the inequality

$$
\left|\left(S_{1}-S_{2}\right) u\right|^{2} \leq \sigma\|u\|^{2}, \quad u \in H .
$$

The quantity $\sigma\left(H_{1}, H_{2}\right)$ is a distance in $\mathcal{S}(H)$-the space of all closed subspaces of $H$. In the next proposition we prove that the Mosco convergence of subspaces (see Sect. 2.3.3 in [10] or Sect.4.5 in [1]) is equivalent to convergence of subspaces with respect to the distance $\sigma$.

\footnotetext{
${ }^{2}$ We note that the spectral problem (19) and (4), and also (20) and (5) have the same eigenvalues and eigenvectors.
} 
Proposition 1. Let $H_{j}, j=1, \ldots$, and $H_{*}$ be subspaces in $H$ and let $S_{j}$ and $S_{*}$ be corresponding orthogonal projectors. the following assertions are equivalent:

(i) for all $u \in H, S_{j} u \rightarrow S_{*} u$ as $j \rightarrow \infty$;

(ii) $\sigma\left(H_{j}, H_{*}\right) \rightarrow 0$ as $j \rightarrow \infty$.

Proof. We denote by $0<s_{1} \leq s_{2} \leq \cdots$, eigenvalues of problem (19), when $H_{1}=H$. Here, we numerate them accounting their multiplicities. Due to the compactness of the imbedding $H \subset \mathcal{H}, s_{k} \rightarrow \infty$ as $k \rightarrow \infty$. We denote by $v_{1}, v_{2}, \ldots$, corresponding eigenvectors which form an orthogonal basis in $H$. We normalize them according to $\left\|v_{k}\right\|=1$. It is clear that

$$
\left(v_{k}, w\right)=s_{k}\left\langle v_{k}, w\right\rangle \text { for all } w \in H .
$$

Therefore, the vectors $w_{k}=\sqrt{s_{k}} v_{k}, k=1,2, \ldots$, form the orthogonal basis in $\mathcal{H}$ subject to $\left|w_{k}\right|=1$.

Let us show that (i) implies (ii). We have

$$
\left(S_{j}-S_{*}\right) u=\sum_{k=1}^{N} s_{k}^{-1 / 2}\left(u,\left(S_{j}-S_{*}\right) v_{k}\right) w_{k}+\sum_{k=N+1}^{\infty} s_{k}^{-1 / 2}\left(\left(S_{j}-S_{*}\right) u, v_{k}\right) w_{k} .
$$

Therefore,

$$
\left|\left(S_{j}-S_{*}\right) u\right|^{2} \leq\left(\sum_{k=1}^{N} s_{k}^{-1}\left\|\left(S_{j}-S_{*}\right) v_{k}\right\|^{2}+s_{N+1}^{-1}\right)\|u\|^{2} .
$$

this implies

$$
\sigma\left(H_{j}, H_{*}\right) \leq \inf _{N}\left(\sum_{k=1}^{N} s_{k}^{-1}\left\|\left(S_{j}-S_{*}\right) v_{k}\right\|^{2}+s_{N+1}^{-1}\right) .
$$

The right hand side in the last inequality tends to zero when $j \rightarrow \infty$ because of strong convergence of $S_{j}$ to $S_{*}$.

Let us prove the implication (ii) $\Rightarrow$ (i). For $u \in H$, we have

$$
\left(\left(S_{j}-S_{*}\right) u, v_{k}\right)=s_{k}\left\langle\left(S_{j}-S_{*}\right) u, v_{k}\right\rangle \rightarrow 0 \text { as } j \rightarrow \infty
$$

for $k=1,2, \ldots$ This implies that $\left(\left(S_{j}-S_{*}\right) u, v\right) \rightarrow 0$ as $j \rightarrow \infty$ for all $v \in H$. Therefore,

$$
\begin{aligned}
& \left(\left(S_{j}-S_{*}\right) u,\left(S_{j}-S_{*}\right) u\right)=\left(S_{j} u, u\right)-\left(S_{*} u, S_{j} u\right)-\left(S_{j} u, S_{*} u\right)+\left(S_{*} h, S_{*} u\right) \\
& \rightarrow\left(S_{*} u, u\right)-\left(S_{*} u, S_{*} u\right)-\left(S_{*} u, S_{*} u\right)+\left(S_{*} h, S_{*} u\right)=0 \text { as } j \rightarrow \infty .
\end{aligned}
$$

The proof is complete. 
Using equivalence of $\gamma$ convergence of domains and strong convergence of corresponding operators (see Theorem 2.3.10 in [9]), we derive from the previous assertion the following

Corollary 1. Let $\Omega_{j}, j=1, \ldots$, and $\Omega_{*}$ be domains belonging to a bounded domain $D$. If $\Omega_{j} \gamma$-converges to $\Omega_{*}$ then $\sigma\left(H_{j}, H_{*}\right) \rightarrow 0$ as $j \rightarrow \infty$, where $H_{j}=\stackrel{\circ}{W}^{1,2}\left(\Omega_{j}\right)$ and $H_{*}=\stackrel{\circ}{W}^{1,2}\left(\Omega_{*}\right)$.

Proposition 2. The metric space $\mathcal{S}(H)$ with the distance $\sigma$ is compact.

Proof. Let $S_{k}, k=1,2, \ldots$, be a family of orthogonal projectors. Let us show that one can choice a convergent subsequence. Let $v_{j}$ be the same vectors as in the proof of Proposition 1. We can choose a subsequence such that

$$
\left(S_{k} v_{i}, v_{j}\right) \rightarrow \alpha_{i j} \quad \text { as } k \rightarrow \infty
$$

for all $i, j \geq 1$. We used the same index for the subsequence in (22). Since $\left\|S_{k}\right\|_{H \rightarrow H}=1$, we derive from (22) that

$$
\left(S_{k} u, v\right) \rightarrow \alpha(u, v) \text { as } k \rightarrow \infty
$$

for all $u, v \in H$, where $\alpha(u, v)$ is a certain number. One can check that the form $\alpha$ is linear with respect to the first argument and anti-linear with respect to the second one. Moreover, the form $\alpha$ is bounded. Therefore, $\alpha(u, v)=\left(S_{*} u, v\right)$, where $S_{*}$ is an orthogonal projector. Reasoning as in the proof of Proposition 1(ii), we conclude that $S_{k} u \rightarrow S_{*} u$ as $k \rightarrow \infty$.

In what follows we shall use that the orthogonal projectors $S_{1}$ and $S_{2}$ posses the following symmetry property:

$$
\left(S_{2} v, w\right)=\left(v, S_{1} w\right) \text { for } v \in H_{1} \text { and } w \in H_{2} \text {. }
$$

\subsection{Formulation of results}

In what follows we denote by $P_{m}$ the orthogonal projector in $H$ with the image $S X_{m}$.

Proposition 3. There exists positive $\sigma_{0}, c$ and $C$ depending on $\lambda_{1}, \ldots, \lambda_{m+1}$ and $c_{0}$ such that for $\sigma \leq \sigma_{0}$ the following assertions are valid:

(i) The operator $K_{2}$ has exactly $J_{m}$ eigenvalue in $\left(1 / \lambda_{m+1}+c \sigma^{1 / 2}, 1 / \lambda_{m-1}-\right.$ $\left.c \sigma^{1 / 2}\right)$ and all of them are located in $\left(1 / \lambda_{m}-c \sigma^{1 / 2}, 1 / \lambda_{m}+c \sigma^{1 / 2}\right)$. 
(ii) If $\mu^{-1}$ is an eigenvalue of (20) from the interval $\left(1 / \lambda_{m}-c \sigma^{1 / 2}, 1 / \lambda_{m}+\right.$ $c \sigma^{1 / 2}$ ) and $U$ is a corresponding eigenvector then

$$
\left\|U-P_{m} U\right\| \leq C \sigma^{1 / 2}\|U\| .
$$

We denote by $\mu_{k}^{-1}, k=1, \ldots, J_{m}$, the eigenvalues of the spectral problem (20) located in the interval $\left(\lambda_{m}^{-1}-c \sigma, \lambda_{m}^{-1}+c \sigma\right)$, where $c$ is the same positive constant as in Proposition 3. In order to formulate the main result of this paper let us introduce some more objects. We put $T_{2} u=u-S_{2} u$ and define the vector $\Psi=\Psi_{\varphi} \in H_{2}$ as the solution of equation (7). Let also

$$
\rho=\max _{\varphi \in X_{m},\|\varphi\|=1}\left(\sigma\left\|\Psi_{\varphi}\right\|^{2}+|T \varphi|^{2}+\left|\Psi_{\varphi}\right|^{2}\right) .
$$

Theorem 2. The following asymptotic formula holds:

$$
\mu_{k}^{-1}=\lambda_{m}^{-1}+\tau_{k}+O\left(\rho+\left|\tau_{k}\right| \sigma\right), \quad k=1, \ldots, J_{m},
$$

where $\tau=\tau_{k}$ is an eigenvalue of the problem

$\frac{1}{\lambda_{m}}\left(\left(\Psi_{\varphi}, \Psi_{\psi}\right)-(T \varphi, T \psi)-\left(\Psi_{\varphi}, \psi\right)-\left(\varphi, \Psi_{\psi}\right)\right)=\tau(S \varphi, S \psi)$ for all $\psi \in X_{m}$

where $\varphi \in X_{m}$. Moreover, $\tau_{1}, \ldots, \tau_{m}$ in (25) run through all eigenvalues of (26) counting their multiplicities.

In Sect. 3.1 we prove that $\|\varphi\|^{2}-\|S \varphi\|^{2} \leq 1-C \sigma^{1 / 2}$ for $\varphi \in X_{m}$. This fact and Theorem 2 lead to the following corollaries.

Corollary 2. If $H_{2} \subset H_{1}$ then $\Psi_{\varphi}=0$ for all $\varphi \in X_{m}$. Therefore, from (26) it follows that $\left|\tau_{k}\right| \leq C \rho$ and hence (25) implies

$$
c \min _{\varphi \in X_{m},\|\varphi\|=1}\|T \varphi\|^{2} \leq\left|\mu_{k}^{-1}-\lambda_{m}^{-1}\right| \leq C \max _{\varphi \in X_{m},\|\varphi\|=1}\|T \varphi\|^{2},
$$

where $c$ and $C$ are positive constants.

Corollary 3. Let $S_{0}$ be the orthogonal projector onto $H_{1} \cap H_{2}$ and let $T_{0} u=$ $u-S_{0} u$. Then $\left(\Psi_{\varphi}, \psi\right)=\left(\Psi_{\varphi}, T_{0} \psi\right)$ and therefore from (26) it follows $\left|\tau_{k}\right| \leq$ $C \rho_{0}$, where

$$
\rho_{0}=\max _{\varphi \in X_{m},\|\varphi\|=1}\left(\left\|T_{0} \varphi\right\|^{2}+\left\|\Psi_{\varphi}\right\|^{2}\right) .
$$

Consequently,

$$
\left|\mu_{k}^{-1}-\lambda_{m}^{-1}\right| \leq C \rho_{0}
$$

for arbitrary $H_{1}$ and $H_{2}$ subject to (21) with small constant $\sigma$. 
Remark 1. In [12] and 14] another quantity is used to measure proximity of two subspaces. I is defined as a constant $\sigma_{*}=\sigma_{*}\left(H_{1}, H_{2}\right)$ in the inequality

$$
|u|^{2} \leq \sigma_{*}\|u\|^{2}
$$

for all elements $u$ from $u \in H_{1}+H_{2}$ orthogonal to $H_{1} \cap H_{2}$. Since

$$
\left|\left(S_{1}-S_{2}\right) u\right| \leq \sqrt{\sigma_{*}}||\left(S_{1}-S_{2}\right) u\left\|\leq 2 \sqrt{\sigma_{*}}|| u\right\|,
$$

the constant $\sigma$ in (21) is subject to

$$
\sigma \leq 4 \sigma_{*} .
$$

One can check that $T \varphi$ and $\Psi_{\varphi}$ belong to $H_{1}+H_{2}$ and both of them are orthogonal to $H_{1} \cap H_{2}$ for $\varphi \in X_{m}$. Therefore, $|T \varphi| \leq\left.\sigma_{*}|| T \varphi\right|^{2}$ and $\left|\Psi_{\varphi}\right| \leq$ $\sigma_{*}\left\|\Psi_{\varphi}\right\|^{2}$ due to (27). So, under assumption (27), we have

$$
\rho \leq c \sigma_{*} \rho_{*}, \quad \rho_{*}=\max _{\varphi \in X_{m},\|\varphi\|=1}\left(\|T \varphi\|^{2}+\left\|\Psi_{\varphi}\right\|^{2}\right) .
$$

Using (29), we see that formula (25) in Theorem 2 implies the following asymptotic formula

$$
\mu_{k}^{-1}=\lambda_{m}^{-1}+\tau_{k}+O\left(\sigma_{*} \rho_{*}+\left|\tau_{k}\right| \sigma_{*}\right), \quad k=1, \ldots, J_{m},
$$

where $\tau=\tau_{k}$ is an eigenvalue of the problem (10). Moreover, $\tau_{1}, \ldots, \tau_{m}$ in (30) run through all eigenvalues of (10) counting their multiplicities.

\section{Proof of Proposition 3}

In what follows, we put $\mathcal{X}_{m}=X_{1}+\cdot+X_{m}$ and by $\mathcal{Y}_{m}$ we denote the orthogonal complement of $S \mathcal{X}_{m}$ in $H_{2}$.

An important role will be played by the operator

$$
B=K_{2} S_{2}-S_{2} K_{1}
$$

In Sect 3.1, we will show in particular, that the norm of $B: H_{1} \rightarrow H_{2}$ is estimated by a constant times $\sigma^{1 / 2}$.

By $c, C, \ldots$ we denote various constants depending on $\lambda_{1}, \cdots, \lambda_{m+1}$ and $c_{0}$. 


\subsection{Some inequalities}

In this section we prove some important estimates, which follow from (21) and which will be used in the proofs of Proposition 3 and Theorem 2 .

Estimates for $S_{2}$. Let us start with the following inequality

$$
\left(1-\Lambda_{m} \sigma^{1 / 2}\right)\|\varphi\|^{2} \leq\|S \varphi\|^{2} \leq\|\varphi\|^{2} \text { for } \varphi \in \mathcal{X}_{m}
$$

where $\Lambda_{m}=\left(\sum_{k=1}^{m} \lambda_{k}\right)^{1 / 2}$. Since $S_{2}$ is an orthogonal projector, the right inequality is obvious. Let us prove the left one. We represent $\varphi$ as $\varphi_{0}+\varphi_{1}$, where $\varphi_{0}=S_{2} \varphi$ and $\varphi_{1}=\left(I-S_{2}\right) \varphi$. Since $S_{2} \varphi_{0}=\varphi_{0}$ and $S_{2} \varphi_{1}=0$, we have $\|S \varphi\|^{2}=\left\|\varphi_{0}\right\|^{2}$. Using that $S_{1} \varphi=\varphi$, we conclude that $\varphi_{1}=\left(S_{1}-S_{2}\right) \varphi$ and from (21) it follows that

$$
\left|\varphi_{1}\right|^{2}=\left|\left(S_{1}-S_{2}\right) \varphi\right|^{2} \leq \sigma\|\varphi\|^{2} .
$$

Representing $\varphi$ as $\varphi=\zeta_{1}+\ldots+\zeta_{m}$, where $\zeta_{k} \in X_{k}$, and noting that $\left(\varphi_{1}, \varphi_{1}\right)=\left(\varphi, \varphi_{1}\right)$, we get

$$
\begin{aligned}
& \left(\varphi_{1}, \varphi_{1}\right)=\sum_{k=1}^{m}\left(\zeta_{k}, \varphi_{1}\right)=\sum_{k=1}^{m} \lambda_{k}\left\langle\zeta_{k}, \varphi_{1}\right\rangle \leq \sum_{k=1}^{m} \lambda_{k}\left|\zeta_{k}\right|\left|\varphi_{1}\right| \\
& \leq \sum_{k=1}^{m} \lambda_{k}^{1 / 2}|| \zeta_{k} \|\left|\varphi_{1}\right| \leq \Lambda_{m}|| \varphi||\left|\varphi_{1}\right|,
\end{aligned}
$$

where we used that $\zeta_{k}$ is an eigenvector of $K_{1}$ corresponding to the eigenvalue $\lambda_{k}^{-1}$. Using the last inequality together with (33), we get

$$
\left(\varphi_{1}, \varphi_{1}\right) \leq \Lambda_{m} \sigma^{1 / 2}(\varphi, \varphi)
$$

Therefore,

$$
\|S \varphi\|^{2}=\left\|\varphi_{0}\right\|^{2}=\|\varphi\|^{2}-\left\|\varphi_{1}\right\|^{2} \geq\left(1-\Lambda_{m} \sigma^{1 / 2}\right)\|\varphi\|^{2},
$$

which implies the left inequality in (32).

From (32) one can derive the estimate

$$
|(S \varphi, S \psi)-(\varphi, \psi)| \leq 3 \Lambda_{m} \sigma^{1 / 2}\left(\|\varphi\|^{2}+\|\psi\|^{2}\right) \text { for } \varphi, \psi \in \mathcal{X}_{m} .
$$

Indeed, introduce the form $b(u, v)=(S \varphi, S \psi)-(\varphi, \psi)$. By (33),

$$
|b(u, u)| \leq \varepsilon\|u\|^{2},
$$


where $\varepsilon=\Lambda_{m} \sigma^{1 / 2}$. Using that

$$
b(\varphi+\psi, \varphi+\psi)=b(\varphi, \varphi)+b(\psi, \psi)+b(\varphi, \psi)+b(\psi, \varphi)
$$

and applying (35) for estimating quadratic terms here we obtain

$$
|b(\varphi, \psi)+b(\psi, \varphi)| \leq 3 \varepsilon\left(\|\varphi\|^{2}+\|\psi\|^{2}\right) .
$$

Similar arguments applied to $b(\varphi+i \psi, \varphi+i \psi)$ give the estimate

$$
|b(\varphi, \psi)-b(\psi, \varphi)| \leq 3 \varepsilon\left(\|\varphi\|^{2}+\|\psi\|^{2}\right)
$$

which together with the previous one leads to (34).

An estimate for the operator $B$. Let us prove that

$$
\|B v\| \leq 2 c_{0} \sigma^{1 / 2}\|v\| \text { for } v \in H_{1} .
$$

For $w \in H_{2}$ we have

$$
(B v, w)=\left(K_{2} S_{2} v, w\right)-\left(S_{2} K_{1} v, w\right)=\left\langle S_{2} v, w\right\rangle-\left\langle v, S_{1} w\right\rangle .
$$

We write $v$ and $w$ as $v=S_{2} v+\left(I-S_{2}\right) v=v_{0}+v_{1}$ and $w=S_{1} w+\left(I-S_{1}\right) w=$ $w_{0}+w_{1}$. Then (37) implies

$$
(B v, w)=\left\langle v_{0}, w_{1}\right\rangle-\left\langle v_{1}, w_{0}\right\rangle
$$

where we have used the equalities $S_{2} v_{1}=S_{1} w_{1}=0$. Since $v_{1}=\left(S_{1}-S_{2}\right) v$ and $w_{1}=\left(S_{2}-S_{1}\right) w$, then by using estimate (21) for function containing index 1 , we get

$$
|(B v, w)| \leq \sigma^{1 / 2}\left(\left|v_{0}\right|\|w\|+\|v\|\left|w_{0}\right|\right) .
$$

Applying here estimate (18), we get

$$
|(B v, w)| \leq 2 c_{0} \sigma^{1 / 2}\|v\|\|w\|
$$

which implies (36).

An inequality for $K_{1}$ and $K_{2}$. Finally, let us show that

$$
\left(K_{2} w, w\right) \leq\left(K_{1} S_{1} w, S_{1} w\right)+\left(2 c_{0} \sqrt{\sigma}+\sigma\right)\|w\|^{2} \quad \text { for } w \in H_{2},
$$


or what is equivalent, due to the definition of $K_{1}$ and $K_{2}$,

$$
|w|^{2} \leq\left|S_{1} w\right|^{2}+\left(2 c_{0} \sqrt{\sigma}+\sigma\right)\|w\|^{2}
$$

We write $w=S_{1} w+\left(I-S_{1}\right) w=w_{0}+w_{1}$. Since $S_{1} w_{1}=0$ and $S_{1} w_{0}=w_{0}$, relation (40) takes the form

$$
\left|w_{0}+w_{1}\right|^{2} \leq\left|w_{0}\right|^{2}+\left(2 c_{0} \sqrt{\sigma}+\sigma\right)|| w||^{2}
$$

or

$$
\left|w_{1}\right|^{2}+2\left\langle w_{0}, w_{1}\right\rangle \leq\left(2 c_{0} \sqrt{\sigma}+\sigma\right)\|w\|^{2} .
$$

Using that $w_{1}=\left(S_{2}-S_{1}\right) w$ and applying (21), we estimate the left-hand side of (41) by

$$
\sigma\|w\|^{2}+2 \sigma^{1 / 2}\left|w_{0}\right|\|w\|
$$

According to (18), $\left|w_{0}\right| \leq c_{0}|| w_{0}\left\|\leq c_{0}\right\| w \|$, which implies (41) and hence (39).

\subsection{Proof of Proposition $3(\mathrm{i})$}

1) Let $u \in \mathcal{Y}_{m}$. Then $S_{1} u$ is orthogonal to $X_{1}+\cdots+X_{m}$ and by (39)

$$
\begin{aligned}
& \left(K_{2} u, u\right) \leq\left(K_{1} S_{1} u, S_{1} u\right)+c \sigma^{1 / 2}\|u\|^{2} \leq \frac{1}{\lambda_{m+1}}\left(S_{1} u, S_{1} u\right)+c \sigma^{1 / 2}\|u\|^{2} \\
& \leq\left(\frac{1}{\lambda_{m+1}}+c \sigma^{1 / 2}\right)\|u\|^{2}
\end{aligned}
$$

From this inequality it follows that there are $\leq J_{1}+\cdots+J_{m}$ eigenvalues of $K_{2}$ counted together with their multiplicity in the interval $\left(1 / \lambda_{m+1}+c \sigma^{1 / 2}, \infty\right)$.

2) Let $u=S_{2} \varphi, \varphi \in \mathcal{X}_{m}$. Then

$$
\left(K_{2} u, u\right)=\left(S_{2} K_{1} \varphi, S_{2} \varphi\right)+(B \varphi, u)
$$

and using (36), we get

$$
\left(K_{2} u, u\right) \geq\left(S_{2} K_{1} \varphi, S_{2} \varphi\right)-C \sigma^{1 / 2}\|\varphi\|\|u\| .
$$

Applying (34) to the first term in the left-hand side, we obtain

$$
\left(K_{2} u, u\right) \geq\left(K_{1} \varphi, \varphi\right)-C_{1} \sigma^{1 / 2}\left(\left\|K_{1} \varphi\right\|^{2}+\|\varphi\|^{2}\right)-C \sigma^{1 / 2}\|\varphi\|\|u\|,
$$


which leads to

$$
\left(K_{2} u, u\right) \geq\left(K_{1} \varphi, \varphi\right)-C_{2} \sigma^{1 / 2}\left(\|\varphi\|^{2} \geq\left(\frac{1}{\lambda_{m-1}}-C_{2} \sigma^{1 / 2}\right)\|\varphi\|^{2} .\right.
$$

Applying (32) for estimating the second term in the right-hand side in the last inequality, we arrive at

$$
\left(K_{2} u, u\right) \geq\left(\frac{1}{\lambda_{m}}-C_{2} \sigma^{1 / 2}\right)\|u\|^{2} .
$$

This implies that there are $\geq J_{1}+\cdots+J_{m}$ eigenvalues of $K_{2}$ in the interval $\left(1 / \lambda_{m}-C_{2} \sigma^{1 / 2}, \infty\right)$.

Combining 1) and 2) we conclude that there are exactly $J_{1}+\cdots+J_{m}$ eigenvalues of $K_{2}$ in the interval $\left(\gamma_{1}, \infty\right)$ for $\gamma_{1} \in\left(1 / \lambda_{m+1}+C_{1} \sigma^{1 / 2}, 1 / \lambda_{m}-\right.$ $\left.C_{2} \sigma^{1 / 2}\right)$.

Applying 1) and 2) with $m$ replaced by $m-1$ we obtain that there are exactly $J_{1}+\cdots+J_{m-1}$ eigenvalues of $K_{2}$ in the interval $\left(\gamma_{2}, \infty\right)$ for $\gamma_{2} \in\left(1 / \lambda_{m}+C_{1} \sigma^{1 / 2}, 1 / \lambda_{m-1}-C_{2} \sigma^{1 / 2}\right)$. Therefore there exists positive constants $c$ and $\sigma_{0}$ depending on $\lambda_{1}, \ldots, \lambda_{m+1}$ and $c_{0}$ such that the intervals $\left(1 / \lambda_{m}-c \sigma^{1 / 2}, 1 / \lambda_{m}+c \sigma^{1 / 2}\right)$ and $\left(1 / \lambda_{m+1}+c \sigma^{1 / 2}, 1 / \lambda_{m-1}-c \sigma^{1 / 2}\right)$ contains exactly $J_{m}$ eigenvalues of $K_{2}$ for $\sigma \leq \sigma_{0}$. The proof of Proposition 3(i) is complete.

\subsection{Proof of Proposition 3(ii)}

First, let us consider the equation

$$
Q_{m} K_{2} w-\mu w=f
$$

where $f, w \in Q_{m} H_{2}, Q_{m}=I-P_{m}$ and

$$
\left|\mu^{-1}-\lambda_{m}^{-1}\right| \leq c \sigma^{1 / 2}
$$

where $c$ is the same constant as in (i). Our first goal is to prove the estimate

$$
\|w\| \leq c_{1}\|f\|
$$

for solutions of equation (43) . Here the constant $c_{1}$ depends on $\lambda_{1}, \cdots, \lambda_{m+1}$ and $c_{0}$. 
We represent $S_{2} \mathcal{X}_{m}$ as $S_{2} X_{m}+Y_{m}$ where $Y_{m}$ is the orthogonal complement to $S_{2} X_{m}$ in $S_{2} \mathcal{X}_{m}$. We introduce the orthogonal projectors $R_{m}$ onto $Y_{m}$ and $\mathcal{T}_{m}$ onto $\mathcal{Y}_{m}$. Then $Q_{m}=R_{m}+\mathcal{T}_{m}$. We write $w=w_{0}+w_{1}$ and $f=f_{0}+f_{1}$, where $w_{0}, f_{0} \in Y_{m}$ and $w_{1}, f_{1} \in \mathcal{Y}_{m}$. Equation (43) can be written as the following system of equations

$$
R_{m} K_{2}\left(w_{0}+w_{1}\right)-\mu w_{0}=f_{0}
$$

and

$$
\mathcal{T}_{m} K_{2} w_{1}-\mu w_{1}=f_{1}-\mathcal{T}_{m} K_{2} w_{0} .
$$

From the second equation we get

$$
\left(K_{2} w_{1}, w_{1}\right)-\mu\left(w_{1}, w_{1}\right)=\left(f_{1}-\mathcal{T}_{m} K_{2} w_{0}, w_{1}\right) .
$$

Using estimate (42), we obtain

$$
\mu\left(w_{1}, w_{1}\right)-\left(\frac{1}{\lambda_{m+1}}+c \sigma^{1 / 2}\right)\left\|w_{1}\right\|^{2} \leq\left(\mathcal{T}_{m} K_{2} w_{0}, w_{1}\right)-\left(f_{1}, w_{1}\right),
$$

which implies that the operator $\mu^{-1}-Q_{m} K_{2}$ is positive definite on $\mathcal{Y}_{m}$, equation (47) is uniquely solvable and its solution satisfies

$$
\left(\mu-\frac{1}{\lambda_{m+1}}-c \sigma^{1 / 2}\right)\left\|w_{1}\right\|_{2} \leq\left\|\mathcal{T}_{m} K_{2} w_{0}\right\|+\left\|f_{1}\right\|
$$

Furthermore, representing $w_{0}$ as $S \varphi_{0}, \varphi_{0} \in \mathcal{X}_{m}$ we have

$$
\mathcal{T}_{m} K_{2} w_{0}=\mathcal{T}_{m} K_{2} S_{2} \varphi_{0}=\mathcal{T}_{m}\left(S_{2} K_{1} \varphi_{0}+B \varphi_{0}\right)=\mathcal{T}_{m} B \varphi_{0}
$$

Therefore, by (36) and (32)

$$
\left\|\mathcal{T}_{m} K_{2} w_{0}\right\| \leq c \sigma^{1 / 2}\left\|\varphi_{0}\right\| \leq c_{1} \sigma^{1 / 2}\left\|w_{0}\right\|
$$

Combining this estimate with (48), we get

$$
\left\|w_{1}\right\| \leq C\left(\left\|f_{1}\right\|+\sigma^{1 / 2}\left\|w_{0}\right\|\right) .
$$

We represent $\varphi_{0}$ as $\varphi^{\prime}+\varphi_{m}$, where $\varphi_{m} \in X_{m}$ and $\varphi^{\prime} \in X_{1}+\cdots+X_{m-1}$. Since $\left(S_{2} \varphi_{0}, S_{2} \varphi_{m}\right)=0$, using (34) we get

$$
\left(\varphi_{0}, \varphi_{m}\right)=\left\|\varphi_{m}\right\|^{2} \leq 3 \lambda_{m}^{1 / 2} \sigma^{1 / 2}\left\|\varphi_{0}\right\|^{2}
$$


Therefore,

$$
\left(K_{2} w_{0}, w_{0}\right)=\left(K_{2} S_{2} \varphi_{0}, S_{2} \varphi_{0}\right)=\left(S_{2} K_{1} \varphi_{0}, S_{2} \varphi_{0}\right)+\left(B \varphi_{0}, S_{2} \varphi_{0}\right)
$$

Using (34) and (36) for estimating the first and second terms in the righthand side of the last relation respectively we get

$$
\left(K_{2} w_{0}, w_{0}\right) \geq\left(K_{1} \varphi_{0}, \varphi_{0}\right)-c \sigma^{1 / 2}\left\|\varphi_{0}\right\|^{2}
$$

where $c$ depends on the eigenvalues $\lambda_{1}, \lambda_{m}$ and the constant $c_{0}$ in (18). Since

$$
\left(K_{1} \varphi_{0}, \varphi_{0}\right)=\left(K_{1} \varphi_{m}, \varphi_{m}\right)+\left(K_{1} \varphi^{\prime}, \varphi^{\prime}\right)=\frac{1}{\lambda_{m}}\left\|\varphi_{m},\right\|^{2}+\left(K_{1} \varphi^{\prime}, \varphi^{\prime}\right)
$$

by using (51), we get

$$
\left(K_{1} \varphi_{0}, \varphi_{0}\right) \geq\left(\frac{1}{\lambda_{m-1}}-c \sigma^{1 / 2}\right)\left\|\varphi_{0}\right\|^{2} \geq\left(\frac{1}{\lambda_{m-1}}-c_{1} \sigma^{1 / 2}\right) \mid w_{0} \|^{2} .
$$

Here we used also (32) in order to obtain the last inequality. Applying the last estimate to the first term in the right-hand side in (52), we obtain

$$
\left(K_{2} w_{0}, w_{0}\right) \geq\left(\frac{1}{\lambda_{m-1}}-c_{2} \sigma^{1 / 2}\right) \mid w_{0} \|^{2}
$$

Using (53) and (49), we conclude that equation (46) is solvable with respect to $w_{0}$ and $\left\|w_{0}\right\| \leq c\|f\|$. Similar estimate for $w_{1}$ follows from (50). Conclusively, equation (43) is uniquely solvable and for its solution $w \in Q_{m} H_{2}$ estimate (45) holds.

Let $\mu^{-1}$ be an eigenvalue of problem (20) satisfying (44) and let $U$ be a corresponding eigenvector. We represent it as $U=S_{2} \varphi_{m}+w$ where $\varphi_{m} \in X_{m}$ and $w \in w \in Q_{m} H_{2}$. Then

$$
K_{2}\left(S_{2} \varphi_{m}+w\right)=\frac{1}{\mu}\left(S_{2} \varphi_{m}+w\right) .
$$

We write this relation as

$$
K_{2} w-\frac{1}{\mu} w+S_{2} K_{1} \varphi_{m}+B \varphi_{m}-\frac{1}{\mu} S_{2} \varphi_{m}=0,
$$


or, equivalently

$$
K_{2} w-\frac{1}{\mu} w=-B \varphi_{m}+\left(\frac{1}{\mu}-\frac{1}{\lambda_{m}}\right) S_{2} \varphi_{m}
$$

We denote the left-hand side by $f$. Then by (36) and assumption on $\mu$, we have

$$
\|f\| \leq C \sigma^{1 / 2}\left\|\varphi_{m}\right\| .
$$

Applying operator $\mathcal{T}_{m}$ to both sides in (54) and using (45) together with the last estimate of $f$ we obtain

$$
\|w\| \leq C \sigma^{1 / 2}\left\|\varphi_{m}\right\| \leq C_{1} \sigma^{1 / 2}\|w\|
$$

\subsection{Corollary of Proposition 3}

Let $Z_{m}$ be the space of eigenvectors of the problem (20) corresponding to eigenvalues located in the interval $\left(1 / \lambda_{m}-c \sigma^{1 / 2}, 1 / \lambda_{m}+c \sigma^{1 / 2}\right)$, see Proposition [3(i). According to the same proposition $\operatorname{dim} Z_{m}=J_{m}$. Let us show that

$$
\left|(U, V)-\left(P_{m} U, P_{m} V\right)\right| \leq C \sigma\left(\|U\|^{2}+\|V\|^{2}\right) \text { for all } U, V \in Z_{m}
$$

First, let us check that

$$
(U, U)-\left(P_{m} U, P_{m} U\right) \leq C \sigma\|U\|^{2} \text { for all } U \in Z_{m} .
$$

Indeed, introduce an orthonormal basis $U_{1}, \ldots, U_{J_{m}}$ in $Z_{m}$ consisting of eigenvectors of problem (20). If $U \in Z_{m}$ we represent it as $U=a_{1} U_{1}+\cdots+a_{J_{m}} U_{J_{m}}$. Using (23), we get

$$
\left\|U-P_{m} U\right\| \leq \sum_{j=1}^{J_{m}}\left|a_{j}\right|\left\|U_{j}-P_{m} U_{j}|| \leq c \sigma^{1 / 2} \sum_{j=1}^{J_{m}}\left|a_{j}\right| \leq c_{1} \sigma^{1 / 2}\right\| U \|,
$$

which implies (57) since $\left\|U-P_{m} U\right\|^{2}=(U, U)-\left(P_{m} U, P_{m} U\right)$.

In order to prove (56) we introduce the quasi-linear form $b(U, V)=$ $(U, V)-\left(P_{m} U, P_{m} V\right)$. Since $b(U, U) \geq 0$, we have

$$
|b(U, V)| \leq b(U, U)^{1 / 2} b(V, V)^{1 / 2} \leq \frac{1}{2}(b(U, U)+b(V, V))
$$

which together with (57) implies (56). 


\section{Proof of Theorem 2}

\subsection{A finite dimensional reduction}

We represent the function $U \in H_{2}$ in (15) as $U=S_{2} \varphi+w$, where $\varphi \in X_{m}$ and $w \in Q_{m} H_{2}$. Then (5) takes the form

$$
\left(\mu^{-1}-K_{2}\right)\left(S_{2} \varphi+w\right)=0 .
$$

By using the operator $B$, we can write the last relation as

$$
\left(\frac{1}{\mu}-\frac{1}{\lambda_{m}}\right) S_{2} \varphi-B \varphi+\left(\mu^{-1}-K_{2}\right) w=0
$$

Applying operators $P_{m}$ and $Q_{m}$ we get

$$
\left(\frac{1}{\mu}-\frac{1}{\lambda_{m}}\right) S_{2} \varphi-P_{m} B \varphi-P_{m} K_{2} w=0
$$

and

$$
Q_{m}\left(\mu^{-1}-K_{2}\right) w=Q_{m} B \varphi .
$$

We assume that $\mu$ satisfies (44). Then the last equation coincides with (43) if we take there $f=-Q_{m} B \varphi$. Therefore equation (59) is uniquely solvable and

$$
\left\|\left(\mu^{-1}-Q_{m} K_{2} Q_{m}\right)^{-1}\right\|_{Q_{m} H_{2} \rightarrow Q_{m} H_{2}} \leq c_{1},
$$

where $c_{1}$ is the constant from (45). Inserting $w=\left(\mu^{-1}-Q_{m} K_{2} Q_{m}\right)^{-1} Q_{m} B \varphi$ in (58), we obtain

$$
\begin{aligned}
& \left(\frac{1}{\mu}-\frac{1}{\lambda_{m}}\right) S_{2} \varphi-P_{m} B \varphi-R(\lambda, \varphi)=0 \\
& R(\lambda, \varphi)=P_{m} K_{2} Q_{m}\left(\mu^{-1}-Q_{m} K_{2} Q_{m}\right)^{-1} Q_{m} B \varphi
\end{aligned}
$$

We represent $R$ as $R(\lambda, \varphi)=\mu P_{m} K_{2} Q_{m} B \varphi+R_{1}(\lambda, \varphi)$, where

$$
R_{1}(\lambda, \varphi)=P_{m} K_{2} Q_{m} K_{2} Q_{m}\left(\mu^{-1}-Q_{m} K_{2} Q_{m}\right)^{-1} Q_{m} B \varphi .
$$

Equation (61) becomes

$$
\left(\frac{1}{\mu}-\frac{1}{\lambda_{m}}\right) S_{2} \varphi-P_{m} B \varphi-\mu P_{m} K_{2} Q_{m} B \varphi-R_{1}(\lambda, \varphi)=0 .
$$


Taking the inner product of the left-hand side with $S_{2} \psi$ in $H_{2}$, where $\psi \in X_{m}$, we get

$\left(\frac{1}{\mu}-\frac{1}{\lambda_{m}}\right)\left(S_{2} \varphi, S_{2} \psi\right)_{2}-\left(B \varphi, S_{2} \psi\right)-\mu\left(Q_{m} B \varphi, K_{2} S_{2} \psi\right)-\left(R_{1}(\lambda, \varphi), S_{2} \psi\right)=0$

Using that $Q_{m} K_{2} S_{2} \psi=Q_{m} B \psi$, we arrive at

$\left(\frac{1}{\mu}-\frac{1}{\lambda_{m}}\right)\left(S_{2} \varphi, S_{2} \psi\right)_{2}-\left(B \varphi, S_{2} \psi\right)_{2}-\mu\left(Q_{m} B \varphi, B \psi\right)_{2}-(L(\mu) B \varphi, B \psi)_{2}=0$,

where $L(\mu)=Q_{m} K_{2} Q_{m}\left(\mu^{-1}-Q_{m} K_{2} Q_{m}\right)^{-1} Q_{m}$. By (60) $)$, one can check the following estimate for the last operator

$$
|(L(\mu) B \psi, B \psi)| \leq C\left(K_{2} Q_{m} B \psi, Q_{m} B \psi\right)_{2}=C\left\langle Q_{m} B \psi, Q_{m} B \psi\right\rangle .
$$

\subsection{Representation of $(B \varphi, S \psi)$}

For $\varphi, \psi \in X_{m}$, we use the representations

$$
\varphi=S_{2} \varphi+T_{2} \varphi, \quad \psi=S_{2} \psi+T_{2} \psi
$$

Then

$$
\begin{aligned}
& \left(B \varphi, S_{2} \psi\right)=\left\langle S_{2} \varphi, S_{2} \psi\right\rangle-\lambda_{m}^{-1}\left(S_{2} \varphi, S_{2} \psi\right) \\
& =\left\langle\varphi-T_{2} \varphi, \psi-T_{2} \psi\right\rangle-\lambda_{m}^{-1}\left(\varphi-T_{2} \varphi, \psi-T_{2} \psi\right)
\end{aligned}
$$

Using the relation

$$
\left(\Psi_{\varphi}, S_{2} \psi\right)=\left(\Psi_{\varphi}, \psi\right)=\left(\varphi, S_{2} \psi\right)-\lambda_{m}\left\langle\varphi, S_{2} \psi\right\rangle
$$

and similar equality with exchanged $\varphi$ and $\psi$, we obtain

$$
\begin{aligned}
& \left(\varphi-T_{2} \varphi, \psi-T_{2} \psi\right)=\left(T_{2} \varphi, T_{2} \psi\right)-(\varphi, \psi)+\left(\varphi, S_{2} \psi\right)+\left(S_{2} \varphi, \psi\right) \\
& =\left(T_{2} \varphi, T_{2} \psi\right)-\lambda_{m}\langle\varphi, \psi\rangle+\left(\Psi_{\varphi}, \psi\right)+\lambda_{m}\left\langle\varphi, S_{2} \psi\right\rangle \\
& +\left(\varphi, \Psi_{\psi}\right)+\lambda_{m}\left\langle S_{2} \varphi, \psi\right\rangle
\end{aligned}
$$

Replacing the last term in (66) according to this formula, we derive from (66)

$$
\left(B \varphi, S_{2} \psi\right)=\left\langle T_{2} \varphi, T_{2} \psi\right\rangle-\lambda_{m}^{-1}\left(\left(T_{2} \varphi, T_{2} \psi\right)+\left(\Psi_{\varphi}, \psi\right)+\left(\varphi, \Psi_{\psi}\right)\right) .
$$


Corollary 4. Let $H_{2} \subset H_{1}$ and let $\lambda_{1}$ be the first eigenvalue of (19) and $\varphi$ be a corresponding eigenfunction. Then $\Psi_{\varphi}=\Psi_{\psi}=0$ and from (63) and (67) it follows that

$$
1-\frac{\lambda_{1}}{\mu_{1}} \leq \frac{\|T \varphi\|^{2}}{\|S \varphi\|^{2}}
$$

where $\mu_{1}$ is the first eigenvalue of (20).

\subsection{Representation of $\left(Q_{m} B \varphi, B \psi\right)$}

For $\varphi \in X_{M}$ and $w \in H_{2}$, we have the following representation

$$
\begin{aligned}
& (B \varphi, w)=\left(K_{2} S_{2} \varphi, w\right)-\frac{1}{\lambda_{m}}\left(S_{2} \varphi, w\right)=\left\langle\varphi-T_{2} \varphi, w\right\rangle-\frac{1}{\lambda_{m}}(\varphi, w) \\
& =-\frac{1}{\lambda_{m}}\left(\Psi_{\varphi}, w\right)-\left\langle T_{2} \varphi, w\right\rangle .
\end{aligned}
$$

This implies

$$
B \varphi=K_{2} \Phi_{\varphi}-\lambda_{m}^{-1} \Psi_{\varphi},
$$

where $\Phi_{\varphi}$ is the orthogonal with respect to the inner product in $\mathcal{H}$ projection of $T_{2} \varphi$ onto $\mathcal{H}_{2}$. Using (69), we get the desired representation

$$
\left(Q_{m} B \varphi, B \psi\right)=\frac{1}{\lambda_{m}^{2}}\left(\Psi_{\varphi}, \Psi_{\psi}\right)+b_{0}(\varphi, \psi),
$$

where

$$
\begin{aligned}
& b_{0}(\varphi, \psi)=-\frac{1}{\lambda_{m}^{2}}\left(P_{m} \Psi_{\varphi}, \Psi_{\psi}\right)+\left(Q_{m} K_{2} \Phi_{\varphi}, K_{2} \Phi_{\psi}\right) \\
& -\frac{1}{\lambda_{m}}\left(\left\langle\Phi_{\varphi}, Q_{m} \Psi_{\psi}\right\rangle+\left\langle Q_{m} \Psi_{\varphi}, \Phi_{\psi}\right\rangle\right) .
\end{aligned}
$$

Let us estimate the form $b_{0}$. Since

$$
\left\|K_{2} \Phi_{\varphi}\right\| \leq C\left|\Phi_{\varphi}\right| \leq C\left|T_{2} \varphi\right|
$$

it follows from (69) and (36) that

$$
\left\|\Psi_{\varphi}\right\| \leq C\left(\sigma^{1 / 2}\|\varphi\|+\left|T_{2} \varphi\right|\right) \text { for } \varphi \in X_{m} .
$$

Using that $T_{2} \varphi=\left(S_{1}-S_{2}\right) \varphi$, we have that $\left|T_{2} \varphi\right|^{2} \leq \sigma\|\varphi\|^{2}$. Therefore, (72) implies

$$
\left\|\Psi_{\varphi}\right\| \leq C \sigma^{1 / 2}\|\varphi\| \text { for } \varphi \in X_{m}
$$


Let $\Upsilon_{k}, k=1, \ldots, J_{m}$, be an orthogonal basis in $S X_{m}$ and let $\Upsilon_{k}=S \varphi_{k}$, $\varphi_{k} \in X_{m}$. Then

$$
\left(P_{m} \Psi_{\varphi}, \Psi_{\psi}\right)=\sum_{k=1}^{J_{m}}\left(\Psi_{\varphi}, S \varphi_{k}\right)\left(S \varphi_{k}, \Psi_{\psi}\right) .
$$

Using definitions of $S, K_{2}$ and $\Psi_{\varphi}$, one can verify that

$$
S \varphi=\lambda_{m} K_{2} \varphi^{*}+\Psi_{\varphi} \quad \text { for } \varphi \in X_{m},
$$

where $\varphi^{*}$ is orthogonal in $\mathcal{H}$ projection of $\varphi$ on $\mathcal{H}_{2}$. Therefore,

$$
\left(\Psi_{\varphi}, S \varphi_{k}\right)=\lambda_{m}\left\langle\Psi_{\varphi}, \varphi_{k}^{*}\right\rangle+\left(\Psi_{\varphi}, \Psi_{\varphi_{k}}\right) .
$$

Applying inequalities (73) for estimation of and $\left\|\Psi_{\varphi_{k}}\right\|$, we get

$$
\left|\left(\Psi_{\varphi}, S \varphi_{k}\right)\right| \leq C\left(\left|\Psi_{\varphi}\right|+\sigma^{1 / 2}|| \Psi_{\varphi}||\right) .
$$

Therefore, it follows from (74) that

$$
\left|\left(P_{m} \Psi_{\varphi}, \Psi_{\psi}\right)\right| \leq C\left(\sigma\left(\left.|| \Psi_{\varphi}\right|^{2}+|| \Psi_{\psi} \|^{2}\right)+\left|\Psi_{\varphi}\right|^{2}+\left|\Psi_{\psi}\right|^{2}\right) .
$$

Since $K_{2}$ is a bounded operator from $\mathcal{H}_{2}$ to $H_{2}$, we have

$$
\left(Q_{m} K_{2} \Phi_{\varphi}, K_{2} \Phi_{\varphi}\right) \leq\left(K_{2} \Phi_{\varphi}, K_{2} \Phi_{\varphi}\right) \leq C\left\langle\Phi_{\varphi}, \Phi_{\varphi}\right\rangle \leq C|T \varphi|^{2} .
$$

Similarly,

$$
\left|\left\langle\Phi_{\varphi}, \Psi_{\psi}\right\rangle+\left\langle\Psi_{\varphi}, \Phi_{\psi}\right\rangle\right| \leq|T \varphi|^{2}+|T \psi|^{2}+\left|\Psi_{\varphi}\right|^{2}+\left|\Psi_{\psi}\right|^{2} .
$$

Combining (76) $-(78)$, we obtain

$$
b_{0}(\varphi, \psi) \leq C(\wp(\varphi)+\wp(\psi)),
$$

where

$$
\wp(\varphi)=\left|T_{2} \varphi\right|^{2}+\left|\Psi_{\varphi}\right|^{2}+\left.\sigma|| \Psi_{\varphi}\right|^{2} .
$$

We note that from the definition of $\rho$, see (24), it follows that

$$
\rho=\max _{\varphi \in X_{m},\|\varphi\|=1} \wp(\varphi) \text {. }
$$




\subsection{Estimate of $\left\langle Q_{m} B \varphi, Q_{m} B \psi\right\rangle$}

Let us consider first the term $\left\langle P_{m} B \varphi, B \psi\right\rangle$. Using the basis $\Upsilon_{k}=S \varphi_{k}$, $k=1, \ldots, J_{m}$, introduced in Sect. 4.3, we have

$$
\left\langle P_{m} B \varphi, B \psi\right\rangle=\sum_{k=1}^{J_{m}}\left(B \varphi, S \varphi_{k}\right)\left\langle S \varphi_{k}, B \psi\right\rangle .
$$

Applying representation (75), we get

$$
\left(B \varphi, S \varphi_{k}\right)=\lambda_{m}\left\langle B \varphi, \varphi_{k}^{*}\right\rangle+\left(B \varphi, \Psi_{\varphi_{k}}\right),
$$

which together with (73) and (69) leads

$$
\left(B \varphi, S \varphi_{k}\right) \leq C\left(\left|T_{2} \varphi\right|+\left|\Psi_{\varphi}\right|+\sigma^{1 / 2}|| \Psi_{\varphi} \|\right)
$$

Similarly,

$$
\left|\left\langle S \varphi_{k}, B \psi\right\rangle\right| \leq C\left(\left|T_{2} \psi\right|+\left|\Psi_{\psi}\right|+\sigma^{1 / 2}|| \Psi_{\psi}||\right) .
$$

Applying these for estimating the right-hand side of (181), we get

$$
\left|\left\langle P_{m} B \varphi, B \psi\right\rangle\right| \leq C(\wp(\varphi)+\wp(\psi)) .
$$

Similar considerations give the estimate

$$
\left|\left\langle P_{m} B \varphi, P_{m} B \psi\right\rangle\right| \leq C(\wp(\varphi)+\wp(\psi)) .
$$

Furthermore, using (69), we get

$$
|\langle B \varphi, B \psi\rangle| \leq C\left(\left|T_{2} \varphi\right|^{2}+\left|T_{2} \psi\right|^{2}+\left|\Psi_{\varphi}\right|^{2}+\left|\Psi_{\psi}\right|^{2}\right) .
$$

Applying (82)-(84) for estimating the right-hand side in (64), we get

$$
|(L(\mu) B \varphi, B \psi)| \leq C(\wp(\varphi)+\wp(\psi)) .
$$

\subsection{Proof of Theorem 2}

Let $\mu$ satisfies (44). We put $\widehat{\tau}=\mu^{-1}-\lambda_{m}^{-1}$. Then $\mu=\lambda_{m}-\lambda_{m}^{2} \widehat{\tau}+O(\sigma)$. Therefore, from (70) and (79) it follows that

$$
\mu\left(Q_{m} B \varphi, B \psi\right)=\frac{1-\lambda_{m} \widehat{\tau}}{\lambda_{m}}\left(\Psi_{\varphi}, \Psi_{\psi}\right)+b_{2}(\varphi, \psi)
$$


where

$$
b_{2}(\varphi, \psi)=\mu b_{0}(\varphi, \psi)+\widehat{\tau} \frac{\lambda_{m}-\mu}{\lambda_{m}}\left(\Psi_{\varphi}, \Psi_{\psi}\right)
$$

and $b_{0}$ is given by (71). Using (79) and (44), we get

$$
b_{2}(\varphi, \psi) \leq C(\wp(\varphi)+\wp(\psi)) .
$$

Using (67), (86) and (87) together with (85), we derive from (63)

$$
\begin{aligned}
& \widehat{\tau}\left((S \varphi, S \psi)+\left(\Psi_{\varphi}, \Psi_{\psi}\right)\right)=\frac{1}{\lambda_{m}}\left(\left(\Psi_{\varphi}, \Psi_{\psi}\right)-(T \varphi, T \psi)\right. \\
& \left.-\left(\Psi_{\varphi}, \psi\right)-\left(\varphi, \Psi_{\psi}\right)\right)+b(\varphi, \psi) \text { for all } \psi \in X_{m},
\end{aligned}
$$

where

$$
b(\varphi, \psi)=b_{2}(\varphi, \psi)+(L(\mu) B \varphi, B \psi)+\left\langle T_{2} \varphi, T_{2} \psi\right\rangle .
$$

Due to (87) and (85), the form $b$ is subject to

$$
|b(\varphi, \psi)| \leq C(\wp(\varphi)+\wp(\psi)) .
$$

Moreover, by (73)

$$
\left|\left(\Psi_{\varphi}, \Psi_{\psi}\right)\right| \leq c \sigma\left(\|\varphi\|^{2}+\|\psi\|^{2}\right) .
$$

From (32) it follows that $\|\varphi\|^{2} \leq\left(1+c \sigma^{1 / 2}\right)\|S \varphi\|^{2}$. Therefore

$$
\left|\left(\Psi_{\varphi}, \Psi_{\psi}\right)\right| \leq c \sigma\left(\|S \varphi\|^{2}+\|S \psi\|^{2}\right) .
$$

Let $\mu_{1}^{-1}, \ldots, \mu_{J_{m}}^{-1}$ be the eigenvalues of the problem (20) and let $U_{1}, \ldots, U_{J_{m}}$ be corresponding eigenvectors. We assume that the eigenvectors are chosen to satisfy $\left(U_{j}, U_{k}\right)=\delta_{j, k}$, where $\delta_{j, k}$ is the Kronecker delta. Since $P_{m}$ is the orthogonal projector with the image $S X_{m}$, we can represent $P_{m} U_{j}$ as $P_{m} U_{j}=S V_{j}$, where $V_{j} \in X_{m}$. According to (56),

$$
\left(S V_{j}, S V_{k}\right)=\delta_{j k}+\mathcal{O}(\sigma) \text { for } j, k=1, \ldots, J_{m} .
$$

Moreover according to the reduction from Sect. 4.1, $\hat{\tau}_{j}=\mu_{j}^{-1}-\lambda_{m}^{-1}, j=$ $1, \cdots, J_{m}$, is an eigenvalue to problem (88) and $\varphi=V_{j}$ is the corresponding eigenvector.

Denote by $\tau_{k}, k=1, \ldots, J_{m}$, the eigenvalue of the finite-dimensional problem (26) and by $\Phi_{1}, \ldots, \Phi_{J_{m}}$ corresponding eigenvectors from $X_{m}$, which satisfies the bi-orthogonality condition

$$
\left(S \Phi_{j}, S \Phi_{k}\right)=\delta_{j k} \quad \text { for } j, k=1, \ldots, J_{m} .
$$


Let us show that for each $j=1, \ldots, J_{m}$ there exists $k=k(j), 1 \leq k \leq \ldots, J_{m}$ such that

$$
\left(S V_{j}, S \Phi_{k(j)}\right) \geq c_{*},
$$

where $c_{*}$ is a positive constant depending on $J_{m}$. Moreover the function $k(j)$ is isomorphism. In order to prove these facts we consider the matrix $A=\left\{A_{j k}\right\}$, where $A_{j k}=\left(S V_{j}, S \Phi_{k}\right), j, k=1, \ldots, J_{m}$. Let us show that

$$
\operatorname{det} A=1+\mathcal{O}\left(\sigma^{1 / 2}\right)
$$

Indeed, let $\nu$ be an eigenvalue of the matrix $A$ and $\mathbf{a}=\left(a_{1}, \ldots, a_{J_{m}}\right)$ be corresponding eigenvector with the norm 1 . Then

$$
\sum_{k}\left(S V_{j}, S \Phi_{k}\right) a_{k}=\nu a_{j} \text { for } j=1, \ldots, J_{m},
$$

or equivalently $\left(S V_{j}, S \Phi\right)=\nu a_{j}$, where $\Phi=\bar{a}_{1} \Phi_{1}+\cdots+\bar{a}_{J_{m}} \Phi_{J_{m}}$. Clearly, $\|S \Phi\|=1$. We chose the constants $b_{j}, j=1, \ldots, J_{m}$, such that $\sum b_{j} V_{j}=\Phi$. Clearly, the norm of the vector $\mathbf{b}=\left(b_{1}, \ldots, b_{J_{m}}\right)$ is equal to $1+\mathcal{O}(\sigma)$. Then

$$
\sum_{j=1}^{J_{m}} b_{j}\left(S V_{j}, S \Phi\right)=(S \Phi, S \Phi)=\nu \sum_{j=1}^{J_{m}} a_{j} b_{j} .
$$

This implies $1 \leq|\nu|(1+\mathcal{O}(\sigma))$. Since the last relation is valid for all eigenvalues of $A$ we obtain (94). Therefore, there exists an isomorphism $k(j)$ such that the equality (93) is valid for $j=1, \cdots, J_{m}$. After the re-numeration of eigenvalues $\tau_{j}$ and corresponding eigenvectors we can assume that the relations

$$
\left(S V_{j}, S \Phi_{j}\right) \geq c_{*}, \quad j=1, \ldots, J_{m},
$$

hold.

Choosing $\varphi=V_{j}$ and $\psi=\Phi_{j}$ in (88) we obtain

$$
\left(\widehat{\tau}_{k}-\tau_{k}\right)\left(S V_{j}, S \Phi_{j}\right)=b\left(V_{j}, \Phi_{j}\right)-\widehat{\tau}_{j}\left(\Psi_{V_{j}}, \Psi_{\Phi_{j}}\right) .
$$

Using relations (95), (89) and (73) together with definition (24) of $\rho$, we derive from (96)

$$
\left|\widehat{\tau}_{k}-\tau_{k}\right| \leq C\left(\max _{\psi \in X_{m},\|\psi\|=1} \wp(\psi)+\left|\widehat{\tau}_{k}\right| \sigma\right) .
$$


By (80), we get

$$
\left|\widehat{\tau}_{k}-\tau_{k}\right| \leq C\left(\rho+\left|\widehat{\tau}_{k}\right| \sigma\right)
$$

This implies

$$
\left|\widehat{\tau}_{k}-\tau_{k}\right| \leq C\left(\rho+\left|\tau_{k}\right| \sigma\right)
$$

and hence (25).

\section{Application to a second order elliptic equa- tion}

Here we consider the spectral problems (44) and (5) generated by the bi-linear form (2). Instead of (21) it is sufficient to check inequality (27).

\subsection{Local perturbation of the boundary}

Constant $\sigma_{*}$. Let $\varepsilon$ be a small positive number. We assume that there exists a point $x_{0}$ in $\partial \Omega_{1}$ such that

$$
\Omega_{1} \backslash B_{\varepsilon}\left(x_{0}\right) \subset \Omega_{2} \subset \Omega_{1} \cup B_{\varepsilon}\left(x_{0}\right) .
$$

We denote the domain $\Omega_{1} \cup B_{\varepsilon}\left(x_{0}\right)$ by $\Omega_{\varepsilon}^{+}\left(x_{0}\right)$. We also assume that for $u \in \stackrel{\circ}{W}^{1,2}\left(\Omega_{\varepsilon}^{+}\left(x_{0}\right)\right)$

$$
\int_{B_{q \varepsilon}\left(x_{0}\right) \cap \Omega_{\varepsilon}^{+}\left(x_{0}\right)}|u|^{2} d x \leq C \varepsilon^{2} \int_{B_{q \varepsilon}\left(x_{0}\right) \cap \Omega_{\varepsilon}^{+}\left(x_{0}\right)}|\nabla u|^{2} d x
$$

for a certain $q>1$ independent of $\varepsilon$. Here the constant $C$ may depend on $\Omega_{1}, n, q$ and the ellipticity constant $\nu$.

Let $\mathcal{Z}_{\varepsilon}\left(x_{0}\right)$ be subspace of function in $\stackrel{\circ}{W}^{1,2}\left(\Omega_{\varepsilon}^{+}\right)$subject to

$$
\sum_{i, j=1}^{n} \int A_{i j}(x) \partial_{x_{j}} u \partial_{x_{i}} w d x=0 \text { for all } w \in \stackrel{\circ}{W}^{1,2}\left(\Omega_{1} \backslash B_{\varepsilon}\left(x_{0}\right)\right) \text {. }
$$

In what follows we will omit the summation sign in formulas like (102).

Lemma 1. (i) Let $u \in \mathcal{Z}_{q^{1 / 2} \varepsilon}\left(x_{0}\right)$. Then

$$
\int_{\Omega_{\varepsilon}^{+}\left(x_{0}\right)}|\nabla u|^{2} d x \leq C_{1} \int_{B_{q \varepsilon}\left(x_{0}\right) \cap\left(\Omega_{\varepsilon}^{+}\left(x_{0}\right)\right.}|\nabla u|^{2} d x .
$$


(ii) Let $q_{*} \in\left[1, q^{1 / 2}\right]$ and let $T_{q_{*} \varepsilon}\left(x_{0}\right)$ be orthogonal projector from $\stackrel{\circ}{W}^{1,2}\left(\Omega_{\varepsilon}^{+}\left(x_{0}\right)\right)$ onto $\mathcal{Z}_{q_{*} \varepsilon}\left(x_{0}\right)$. Then

$$
\int_{\Omega_{\varepsilon}^{+}\left(x_{0}\right)}\left|\nabla\left(T_{q_{*} \varepsilon}\left(x_{0}\right) u\right)\right|^{2} d x \leq C_{1} \int_{B_{q \varepsilon}\left(x_{0}\right) \cap\left(\Omega_{\varepsilon}^{+}\left(x_{0}\right)\right.}|\nabla u|^{2} d x .
$$

The constant $C_{1}$ in (i) and (ii) may depend on $n, \nu, q$ and $\Omega_{1}$.

Proof. We introduce a smooth function $\eta=\eta(t)$ which is equal to 1 for $t<q^{1 / 2}$ and to 0 for $t>q$ and let $\eta_{\varepsilon}(x)=\eta\left(\left|x-x_{0}\right| / \varepsilon\right)$.

(i) Put $u_{\varepsilon}=u-\eta_{\varepsilon} u$. One can check that $u_{\varepsilon} \in W^{1,2}\left(\Omega_{1} \backslash B_{q^{1 / 2} \varepsilon}\left(x_{0}\right)\right)$. From (102) it follows that

$$
\int A_{i j} \partial_{x_{j}} u_{\varepsilon} \partial_{x_{j}} w d x=-\int A_{i j} \partial_{x_{j}}\left(\eta_{\varepsilon} u\right) \partial_{x_{i}} w d x \quad \text { for all } w \in \stackrel{\circ}{W}^{1,2}\left(\Omega_{1} \backslash B_{q^{1 / 2} \varepsilon}\left(x_{0}\right)\right) \text {. }
$$

Taking $w=u_{\varepsilon}$ in (105) we obtain

$$
\int A_{i j} \partial_{x_{j}} u_{\varepsilon} \partial_{x_{i}} u_{\varepsilon} d x=-\int A_{i j} \partial_{x_{j}}\left(\eta_{\varepsilon} u\right) \partial_{x_{i}} u_{\varepsilon} d x
$$

which together with (3) implies

$$
\int\left|\nabla u_{\varepsilon}\right|^{2} d x \leq \nu^{-4} \int\left|\nabla\left(\eta_{\varepsilon} u\right)\right|^{2} d x
$$

Using (101), we derive the following estimate from the last inequality:

$$
\int_{\Omega_{1}}\left|\nabla u_{\varepsilon}\right|^{2} d x \leq C_{1} \int_{B_{q \varepsilon}\left(x_{0}\right) \cap\left(\Omega_{\varepsilon}^{+}\left(x_{0}\right)\right.}|\nabla u|^{2} d x .
$$

Similar estimate for $\eta_{\varepsilon} u$ follows from (101), which together with the estimate for $u_{\varepsilon}$ leads to (103).

(ii) Let $U_{\varepsilon}=T_{q_{*} \varepsilon}\left(x_{0}\right) u-\eta_{\varepsilon} u$. Then $U_{\varepsilon}(x) \in \stackrel{\circ}{W}^{1,2}\left(\Omega_{1} \backslash B_{q_{*} \varepsilon}\left(x_{0}\right)\right)$ and $U_{\varepsilon}+\eta_{\varepsilon} u \in \mathcal{Z}_{q_{*} \varepsilon}\left(x_{0}\right)$. Therefore,

$$
\int A_{i j} \partial_{x_{j}} U_{\varepsilon} \partial_{x_{j}} w d x=-\int A_{i j} \partial_{x_{j}}\left(\eta_{\varepsilon} u\right) \partial_{x_{j}} w d x \text { for all } w \in \stackrel{\circ}{W^{1,2}}\left(\Omega_{1} \backslash B_{q_{*} \varepsilon}\left(x_{0}\right)\right) \text {. }
$$

Taking here $w=U_{\varepsilon}$ and using Hölder inequality along with inequalities (3), we obtain

$$
\int\left|\nabla U_{\varepsilon}\right|^{2} d x \leq \nu^{-4} \int\left|\nabla\left(\eta_{\varepsilon} u\right)\right|^{2} d x \leq C \int_{B_{q \varepsilon}\left(x_{0}\right) \cap\left(\Omega_{\varepsilon}^{+}\left(x_{0}\right)\right.}|\nabla u|^{2} d x .
$$


To get the last inequality we applied (101). The estimate of $\eta_{\varepsilon} u$ by the righthand side of the last inequality follows from (101). Since $T_{q_{*} \varepsilon}\left(x_{0}\right) u=\eta_{\varepsilon} u+U_{\varepsilon}$, the above two estimates give (104). The proof is complete.

Now we are in position to prove the following

Proposition 4. There exists a function $\sigma_{*}=\sigma_{*}(\varepsilon)$ such that $\sigma_{*}(\varepsilon) \rightarrow 0$ as $\varepsilon \rightarrow 0$ and for all $u \in \mathcal{Z}_{\varepsilon}\left(x_{0}\right)$

$$
\int_{\Omega_{\varepsilon}^{+}\left(x_{0}\right)}|u|^{2} d x \leq \sigma \int_{\Omega_{\varepsilon}^{+}\left(x_{0}\right)}|\nabla u|^{2} d x .
$$

Proof. Let $\lambda_{k}, k=1, \ldots$, be eigenvalues of the problem (4) and let $\varphi_{k}$ be corresponding eigenfunctions normalized by $\|\varphi\|_{W^{1,2}\left(\Omega_{1}\right)}=1$. We represent $u$ as $u=\eta_{q^{-1 / 2} \varepsilon} u+u_{\varepsilon}$. Then $u_{\varepsilon} \in \stackrel{\circ}{W}^{1,2}\left(\Omega_{1}\right)$ and $T_{q^{1 / 2} \varepsilon} u_{\varepsilon}=u_{\varepsilon}$. Therefore we may represent $u$ as

$$
u_{\varepsilon}=\sum_{k=N+1}^{\infty}\left(u_{\varepsilon}, \varphi_{k}\right) \varphi_{k}+\sum_{k=1}^{N}\left(u_{\varepsilon}, T_{q^{1 / 2} \varepsilon} \varphi_{k}\right) \varphi_{k}
$$

Since $\left\{\varphi_{k}\right\}_{k \geq 1}$ is an orthogonal basis in $L^{2}\left(\Omega_{1}\right)$ also and $\left\|\varphi_{k}\right\|_{L^{2}\left(\Omega_{1}\right)}^{2}=\lambda_{k}^{-1}$, we have

$$
\begin{aligned}
& \left\|u_{\varepsilon}\right\|_{L^{2}\left(\Omega_{1}\right)}^{2} \leq \frac{1}{\lambda_{N+1}}\left\|u_{\varepsilon}\right\|_{W^{1,2}\left(\Omega_{1}\right)}^{2}+\sum_{k=1}^{N} \frac{1}{\lambda_{k}}\left|\left(u_{\varepsilon}, T_{q^{1 / 2} \varepsilon} \varphi_{k}\right)\right|^{2} \\
& \leq\left(\frac{1}{\lambda_{N+1}}+\sum_{k=1}^{N} \frac{1}{\lambda_{k}}\left\|T_{q^{1 / 2} \varepsilon} \varphi_{k}\right\|_{W^{1,2}\left(\Omega_{1}\right)}^{2}\right)\left\|u_{\varepsilon}\right\|_{W^{1,2}\left(\Omega_{1}\right)}^{2} .
\end{aligned}
$$

Using (103) we get

$$
\left\|u_{\varepsilon}\right\|_{L^{2}\left(\Omega_{1}\right)}^{2} \leq\left(\frac{1}{\lambda_{N+1}}+C \sum_{k=1}^{N} \frac{1}{\lambda_{k}} \int_{\Omega_{1} \backslash \Omega_{q \varepsilon}}\left|\nabla \varphi_{k}\right|^{2} d x\right)\left\|u_{\varepsilon}\right\|_{W^{1,2}\left(\Omega_{1}\right)}^{2}
$$

Let

$$
\sigma_{1}=\inf \left(\frac{1}{\lambda_{N+1}}+C \sum_{k=1}^{N} \frac{1}{\lambda_{k}} \int_{\Omega_{1} \backslash \Omega_{q \varepsilon}}\left|\nabla \varphi_{k}\right|^{2} d x\right)
$$


then $\sigma_{1}(\varepsilon) \rightarrow 0$ as $\varepsilon \rightarrow 0$ and by the last inequality for $u_{\varepsilon}$ combined with (101), we obtain

$$
\left\|u_{\varepsilon}\right\|_{L^{2}\left(\Omega_{1}\right)}^{2} \leq \sigma_{1}\left\|\nabla\left(u-\eta_{q^{-1 / 2} \varepsilon} u\right)\right\|_{W^{1,2}\left(\Omega_{\varepsilon}^{+}\right)} \leq C_{1}\left(\sigma_{1}+\varepsilon^{2}\right)\|\nabla u\|_{W^{1,2}\left(\Omega_{\varepsilon}^{+}\right)}^{2} .
$$

Using (101) again, we get

$$
\left\|\eta_{q^{-1 / 2} \varepsilon} u\right\|_{L^{2}\left(\Omega_{\varepsilon}^{+}\left(x_{0}\right)\right)}^{2} \leq C_{2} \varepsilon^{2} \int_{\Omega_{\varepsilon}^{+}\left(x_{0}\right) \backslash B_{q \varepsilon}\left(x_{0}\right)}|\nabla u|^{2} d x .
$$

Now setting $\sigma=2 C_{1}\left(\sigma_{1}+\varepsilon\right)+2 C_{2} \varepsilon^{2}$ and using the representation $u=$ $\eta_{q^{-1 / 2} \varepsilon} u+u_{\varepsilon}$ we arrive at (107).

Remark 2. In the case $\Omega_{2} \subset \Omega_{1}$ Lemma 1 and Proposition 4 are valid if $\Omega_{\varepsilon}\left(x_{0}\right)$ is replaced by $\Omega_{1}$ in their formulations and in (101).

Remark 3. Let several points $x_{1}, \ldots, x_{m}$ be given on the boundary $\partial \Omega_{1}$. Let also $\varepsilon$ be a small positive number, $q>1$ and inequality (101) be valid for all points $x_{1}, \ldots, x_{m}$. Then Lemma 1 and Proposition 4 remain true for perturbation $\Omega_{2}$ subject to

$$
\Omega_{1} \backslash \bigcup_{j=1}^{m} B_{\varepsilon}\left(x_{j}\right) \subset \Omega_{2} \subset \Omega_{1} \cup \bigcup_{j=1}^{m} B_{\varepsilon}\left(x_{j}\right)
$$

if we replace $\Omega_{\varepsilon}^{+}\left(x_{0}\right)$ by $\Omega_{1} \cup \bigcup_{j=1}^{m} B_{\varepsilon}\left(x_{j}\right)$ and $B_{q \varepsilon}\left(x_{0}\right)$ by $\bigcup_{j=1}^{m} B_{q \varepsilon}\left(x_{j}\right)$ in their formulations.

Estimates of the function $u=T \varphi, \varphi \in X_{m}$. Since $T T_{\varepsilon}=T_{\varepsilon} T=T$, we have that

$$
\int A_{i j} \partial_{x_{j}}(T \varphi) \partial_{x_{i}}(T \varphi) d x \leq \int A_{i j} \partial_{x_{j}}\left(T_{\varepsilon} \varphi\right) \partial_{x_{i}}\left(T_{\varepsilon} \varphi\right) d x
$$

This together with Lemma 1(ii) gives

$$
\|\nabla(T \varphi)\|_{L^{2}\left(\Omega_{2}\right)} \leq c\|\nabla(T \varphi)\|_{L^{2}\left(B_{q \varepsilon}\left(x_{0}\right) \cap \Omega_{\varepsilon}^{+}\right)} .
$$

Estimate of the function $\Psi=\Psi_{\varphi}$. We seek $\Psi$ in the form $\Psi=\eta_{\varepsilon}(x)+v$, where $\eta=\eta(t)$ is a smooth function equals 1 for $t<q_{1}=(1+q) / 2$ and 0 for $t>q_{2}=\left(1+q_{1}\right) / 2$. Then the function $v$ belongs to $\stackrel{\circ}{W}^{1,2}\left(\Omega_{2}\right)$ and satisfies

$$
(v, w)=-\lambda_{m}\left\langle\varphi, \eta_{\epsilon} w\right\rangle+\int_{\Omega_{2}} A_{i j} \partial_{x_{j}} v w \partial_{x_{i}} \eta_{\epsilon} d x-\int_{\Omega_{2}} v A_{i j} \partial_{x_{j}} \eta_{\varepsilon} \partial_{x_{i}} w d x
$$


Applying Hölder's inequality to the left-hand side of (110) and using then (101) we arrive at

$$
\|\nabla v\|_{L^{2}\left(\Omega_{2}\right)} \leq c\|\nabla \varphi\|_{L^{2}\left(\Omega_{1} \cap B_{q \delta}\left(x_{2}\right)\right)} .
$$

Applying (101), we get an estimate of $\eta_{\varepsilon}$ by the right-hand side of (111). Combining these two estimates, we obtain

$$
\|\nabla \Psi\|_{L^{2}\left(\Omega_{2}\right)} \leq c \mid\|\nabla \varphi\|_{L^{2}\left(\Omega_{1} \cap B_{q \varepsilon}\left(x_{0}\right)\right)} .
$$

Using estimates (109), (112) and Corollary 3, we arrive at

Corollary 5. Under the assumptions on $\Omega_{1}$ and $\Omega_{2}$ of this section, the following estimate for the eigenvalues of the problems (4) and (5) holds

$$
\left|\lambda_{m}^{-1}-\mu_{k}^{-1}\right| \leq C \max _{\varphi \in X_{m},\|\varphi\|=1}\|\nabla \varphi\|_{L^{2}\left(\Omega_{1} \cap B_{q \varepsilon}\left(x_{0}\right)\right)}^{2}
$$

where $\mu_{1}, \ldots, \mu_{J_{m}}$ are eigenvalues of (5) located near $\lambda_{m}^{-1}$, see Proposition 3 .

\subsection{Global perturbation of the boundary}

Here we consider perturbations of $\Omega_{1}$ located near the boundary. Let $\varepsilon$ be a small positive number. We introduce the sets

$$
\Omega_{\varepsilon}=\left\{x \in \Omega_{1}: \operatorname{dist}\left(x, \partial \Omega_{1}\right)>\varepsilon\right\} \text { and } \Omega_{\varepsilon}^{+}=\left\{y \in \mathbb{R}^{n}: \operatorname{dist}\left(y, \Omega_{1}\right)<\varepsilon\right) .
$$

We assume that

$$
\Omega_{\varepsilon} \subset \Omega_{2} \subset \Omega_{\varepsilon}^{+}
$$

and that for all $x_{0} \in \partial \Omega_{1}$ and $u \in \stackrel{\circ}{W}^{1,2}\left(\Omega_{\varepsilon}^{+}\right)$the inequality

$$
\int_{B_{q \varepsilon}\left(x_{0}\right) \cap \Omega_{\varepsilon}^{+}}|u|^{2} d x \leq C \varepsilon^{2} \int_{B_{q \varepsilon}\left(x_{0}\right) \cap \Omega_{\varepsilon}^{+}}|\nabla u|^{2} d x
$$

holds with a certain $q>1$ independent of $\varepsilon$.

Let $\mathcal{Z}_{\delta}$ be subspace of function in $\stackrel{\circ}{W}^{1,2}\left(\Omega_{\varepsilon}^{+}\right)$subject to

$$
\sum_{i, j=1}^{n} \int A_{i j}(x) \partial_{x_{j}} u \partial_{x_{i}} w d x=0 \text { for all } w \in \stackrel{\circ}{W}^{1,2}\left(\Omega_{\varepsilon}^{+} \backslash \Omega_{\delta}\right) .
$$


Lemma 2. (i) Let $q_{1} \in(1, q)$ and $u \in \stackrel{\circ}{W}^{1,2}\left(\Omega_{\varepsilon}^{+}\right)$. Then

$$
\int_{\Omega_{\varepsilon}^{+} \backslash \Omega_{q_{1} \varepsilon}}|\nabla u|^{2} d x \leq C_{1} \varepsilon^{2} \int_{\Omega_{\varepsilon}^{+} \backslash \Omega_{q \varepsilon}}|\nabla u|^{2} d x .
$$

(ii) Let $u \in \mathcal{Z}_{q_{0} \varepsilon}$ with $q_{0} \in(1, q)$. Then

$$
\int_{\Omega_{\varepsilon}^{+}}|\nabla u|^{2} d x \leq C_{1} \int_{\Omega_{\varepsilon}^{+} \backslash \Omega_{q \varepsilon}}|\nabla u|^{2} d x .
$$

(iii) Let $q_{*} \in[1, q)$ and let $T_{q_{*} \varepsilon}$ be orthogonal projector from $\stackrel{\circ}{W}^{1,2}\left(\Omega_{\varepsilon}^{+}\right)$ onto $\mathcal{Z}_{q_{*} \varepsilon}$. Then

$$
\int_{\Omega_{\varepsilon}^{+}}\left|\nabla\left(T_{q_{*} \varepsilon} u\right)\right|^{2} d x \leq C_{1} \int_{\Omega_{\varepsilon}^{+} \backslash \Omega_{q \varepsilon}}|\nabla u|^{2} d x,
$$

The constant $C_{1}$ in (i) -(ii) may depend on $n, \nu, q, q_{0}, q_{1}, q_{*}$ and $\Omega_{1}$.

Proof. (i) Let us construct a set of points on $\partial \Omega_{1}$ satisfying the properties a) and b) below. We put $\alpha=\sqrt{q^{2}-q_{1}^{2}}$ and choose points $x_{1}, \ldots, x_{N}$ in the following way. We take an arbitrary point $x_{1} \in \partial \Omega_{1}$. Let the points $x_{1}, \ldots, x_{m}$ have been chosen. If there is a point on the boundary, say $x_{*}$, such that $\left|x-x_{*}\right|>\alpha \varepsilon$ then we put $x_{m+1}=x_{*}$. If there are no such point the the required set is constructed and we take $N=m$. The above procedure leads to a finite set of points with the following properties:

a). $\Omega_{\varepsilon}^{+} \backslash \Omega_{q_{1} \varepsilon} \subset \bigcup_{k=1}^{N} B_{q \varepsilon}\left(x_{k}\right)$,

b). There is a integer $M$, depending only on $n$ and $q_{1}, q$, such that every $x \in \mathbb{R}^{n}$ may belong at most to $M$ balls $B_{q \varepsilon}\left(x_{k}\right), k=1, \ldots, N$.

Using a), (101) and then b), we get

$$
\begin{aligned}
& \int_{\Omega_{\varepsilon}^{+} \backslash \Omega_{q_{1} \varepsilon}}|u|^{2} d x \leq \int_{\bigcup_{k=1}^{N} B_{q \varepsilon}\left(x_{k}\right)}|u|^{2} d x \\
& \leq C \varepsilon^{2} \int_{\bigcup_{k=1}^{N} B_{q \varepsilon}\left(x_{k}\right)}|\nabla u|^{2} d x \leq C M \int_{\Omega_{\varepsilon}^{+} \backslash \Omega_{q \varepsilon}}|\nabla u|^{2} d x,
\end{aligned}
$$

which leads to (116).

(ii). Let $\eta=\eta(t)$ be a smooth function which is equal to 1 for $t<q_{0}$ and 0 for $t>q_{1}=\left(q_{0}+q\right) / 2$. It is clear that $1<q_{0}<q_{1}<q$. Let 
also $\zeta_{\varepsilon}(x)=\eta(d(x) / \varepsilon)$, where $d(x)=\max _{k}\left|x-x_{k}\right|$. We represent $u$ as $u=\zeta_{\varepsilon} u+u_{\varepsilon}$. Then $u_{\varepsilon}$ belongs to $\stackrel{\circ}{W}^{1,2}\left(\Omega_{\varepsilon}^{+} \backslash \Omega_{q_{1} \varepsilon}\right)$ and satisfies

$$
\sum_{i, j=1}^{n} \int A_{i j}(x) \partial_{x_{j}}\left(u_{\varepsilon}+\zeta_{\varepsilon} u\right) \partial_{x_{i}} w d x=0 \quad \text { for all } w \in \stackrel{\circ}{W}^{1,2}\left(\Omega_{\varepsilon}^{+} \backslash \Omega_{q_{0} \varepsilon}\right) .
$$

Taking here $w=u_{\varepsilon}$, moving term with $u$ to the right-hand side, using then Hölder inequality and (3), we obtain

$$
\int\left|u_{\varepsilon}\right|^{2} d x \leq \nu^{-4} \int\left|\nabla\left(\zeta_{\varepsilon} u\right)\right|^{2} d x
$$

which implies, due to (116),

$$
\int_{\Omega_{\varepsilon}^{+}}\left|\nabla u_{\varepsilon}\right|^{2} d x \leq C_{1} \int_{\Omega_{\varepsilon}^{+} \backslash \Omega_{q \varepsilon}}|\nabla u|^{2} d x
$$

The estimate of $\zeta_{\varepsilon} u$ by the left-hand side of (119) follows from (116), which together with (119) gives (117).

(iii) Let $\eta=\eta(t)$ be a smooth function which is equal to 1 for $t<q_{0}=$ $\left(q_{*}+q\right) / 2$ and 0 for $t>q_{1}=\left(q_{0}+q\right) / 2$. One can check that $1<q_{0}<q_{1}<q$. Let also $\zeta_{\varepsilon}(x)=\eta(d(x) / \varepsilon)$. We represent $T_{q_{*} \varepsilon} u$ as $T_{q_{*} \varepsilon} u=\eta_{\varepsilon} u+u_{\varepsilon}$. Since $T_{q_{*} \varepsilon} u(x)=u(x)$ for $x \in \Omega_{\varepsilon}^{+} \backslash \Omega_{q_{*} \varepsilon}$ and

$$
\left(T_{q_{*} \varepsilon} u, w\right)=0 \text { for all } w \in \stackrel{\circ}{W}^{1,2}\left(\Omega_{q_{*} \varepsilon}\right),
$$

we have that $u_{\varepsilon} \in \stackrel{\circ}{W}^{1,2}\left(\Omega_{q_{*} \varepsilon}\right)$ and

$$
\left(u_{\varepsilon}, w\right)=-\left(\zeta_{\varepsilon} u, w\right) \text { for all } w \in \stackrel{\circ}{W}^{1,2}\left(\Omega_{\varepsilon}\right) .
$$

We choose here $w=u_{\varepsilon}$ and obtain

$$
\left\|u_{\varepsilon}\right\|^{2}=-\left(\zeta_{\varepsilon} u, u_{\varepsilon}\right) .
$$

This implies

$$
\left\|u_{\varepsilon}\right\|_{1}^{2} \leq\left\|\zeta_{\varepsilon} u\right\|_{1}^{2}
$$

which leads to

$$
\int_{\Omega_{\varepsilon}^{+}}\left|\nabla u_{\varepsilon}\right|^{2} d x \leq C_{1} \int_{\Omega_{\varepsilon}^{+} \backslash \Omega_{q \varepsilon}}|\nabla u|^{2} d x
$$

where we used (114). Similar estimate of $\eta_{\varepsilon} u$ by the right-hand side of the last inequality follows from (114). These two estimates give (118). 
Proposition 5. There exists a function $\sigma=\sigma$ (varesilon) such that $\sigma(\varepsilon) \rightarrow 0$ as $\varepsilon \rightarrow 0$ and for all $u \in \stackrel{\circ}{W}^{1,2}\left(\Omega_{\varepsilon}^{+}\right)$satisfying (115)

$$
\int_{\Omega_{\varepsilon}^{+}}|u|^{2} d x \leq \sigma \int_{\Omega_{\varepsilon}^{+}}|\nabla u|^{2} .
$$

Proof. Let $\lambda_{k}, k=1, \ldots$, be eigenvalues of the problem (4) and let $\varphi_{k}$ be corresponding eigenfunctions normalized by $\|\varphi\|_{1}=1$. We introduce a smooth $\eta=\eta(t)$ which is equal to 1 for $t<q_{0}=(1+q) / 2$ and 0 for $t>q_{1}=\left(q_{0}+q\right) / 2$. One can check that that $1<q_{0}<q_{1}<q$. Let also $\zeta_{\varepsilon}(x)=\eta(d(x) / \varepsilon)$. We represent $u$ as $u=\eta_{\varepsilon} u+u_{\varepsilon}$. Then $u_{\varepsilon}(x) \in \stackrel{\circ}{W}^{1,2}\left(\Omega_{q_{0} \varepsilon}\right)$ and $T_{q_{1} \varepsilon} u_{\varepsilon}=u_{\varepsilon}$. Therefore, we may represent $u_{\varepsilon}$ as

$$
u_{\varepsilon}=\sum_{k=N+1}^{\infty}\left(u_{\varepsilon}, \varphi_{k}\right) \varphi_{k}+\sum_{k=1}^{N}\left(u_{\varepsilon}, T_{q_{1} \varepsilon} \varphi_{k}\right) \varphi_{k} .
$$

Since $\left\{\varphi_{k}\right\}_{k \geq 1}$ is an orthogonal basis in $L^{2}\left(\Omega_{1}\right)$ also and $\left\|\varphi_{k}\right\|_{L^{2}\left(\Omega_{1}\right)}^{2}=\lambda_{k}^{-1}$, we have

$$
\begin{aligned}
& \left\|u_{\varepsilon}\right\|_{L^{2}\left(\Omega_{1}\right)}^{2} \leq \frac{1}{\lambda_{N+1}}\left\|u_{\varepsilon}\right\|_{W^{1,2}\left(\Omega_{1}\right)}^{2}+\sum_{k=1}^{N} \frac{1}{\lambda_{k}}\left|\left(u_{\varepsilon}, T_{q_{1} \varepsilon} \varphi_{k}\right)\right|^{2} \\
& \leq\left(\frac{1}{\lambda_{N+1}}+\sum_{k=1}^{N} \frac{1}{\lambda_{k}}\left\|T_{q_{1} \varepsilon} \varphi_{k}\right\|_{W^{1,2}\left(\Omega_{1}\right)}^{2}\right)\left\|u_{\varepsilon}\right\|_{W^{1,2}\left(\Omega_{1}\right)}^{2} .
\end{aligned}
$$

Using Lemma 2(ii) we get

$$
\left\|u_{\varepsilon}\right\|_{L^{2}\left(\Omega_{1}\right)}^{2} \leq\left(\frac{1}{\lambda_{N+1}}+C \sum_{k=1}^{N} \frac{1}{\lambda_{k}} \int_{\Omega_{1} \backslash \Omega_{q \varepsilon}}\left|\nabla \varphi_{k}\right|^{2} d x\right)\left\|u_{\varepsilon}\right\|_{W^{1,2}\left(\Omega_{1}\right)}^{2}
$$

Let

$$
\sigma_{1}=2 \inf \left(\frac{1}{\lambda_{N+1}}+C \sum_{k=1}^{N} \frac{1}{\lambda_{k}} \int_{\Omega_{1} \backslash \Omega_{q \varepsilon}}\left|\nabla \varphi_{k}\right|^{2} d x\right) .
$$

then $\sigma_{1}(\varepsilon) \rightarrow 0$ as $\varepsilon \rightarrow 0$ and by the last inequality for $u_{\varepsilon}$, we obtain

$$
\left\|u_{\varepsilon}\right\|_{L^{2}\left(\Omega_{1}\right)}^{2} \leq \sigma_{1}|| \nabla u_{\varepsilon} \|_{W^{1,2}\left(\Omega_{\varepsilon}^{+}\right)} .
$$

Since $u_{\varepsilon}=\left(1-\eta_{\varepsilon}\right) u$, we get

$$
\left\|u_{\varepsilon}\right\|_{L^{2}\left(\Omega_{\varepsilon}^{+}\right)}^{2} \leq C\|\nabla u\|_{W^{1,2}\left(\Omega_{\varepsilon}^{+}\right)} .
$$


Therefore

$$
\left\|u_{\varepsilon}\right\|_{L^{2}\left(\Omega_{1}\right)}^{2} \leq C \sigma_{1}\left\|\nabla u_{\varepsilon}\right\|_{W^{1,2}\left(\Omega_{\varepsilon}^{+}\right)} .
$$

From Lemma 2(i) it follows that

$$
\left\|\eta_{\varepsilon} u\right\|_{L^{2}\left(\Omega_{\varepsilon}^{+}\right)}^{2} \leq C_{1} \varepsilon^{2} \int_{\Omega_{\varepsilon} \backslash \Omega_{q \varepsilon}^{+}}|\nabla u|^{2} d x .
$$

Now setting $\sigma=C \sigma_{1}+C_{1} \varepsilon^{2}$ and using the representation $u=\eta_{\varepsilon} u+u_{\varepsilon}$ we arrive at (120).

Estimates for $T \varphi$. Since $T T_{\varepsilon}=T_{\varepsilon} T=T$, we conclude that $T$ and $T_{\varepsilon}$ satisfy (108). Now, Lemma 2 and (108) lead to the estimate

$$
\|T \varphi\|_{H^{1}\left(\Omega_{2}\right)} \leq C\|\nabla \varphi\|_{L^{2}\left(\Omega_{1} \backslash \Omega_{q \varepsilon}\right.} .
$$

Estimate for $\Psi_{\varphi}, \varphi \in X_{m}$. Let $d=d(x)$ and $\eta_{\varepsilon}(x)=\eta(d(x) / \varepsilon)$ be the same as before. We are looking for the solution $\Psi=\Psi_{\varphi}$ to equation (7) in the form

$$
\Psi=\eta_{\varepsilon}(x) \varphi(x)+v(x) .
$$

Then $v \in \stackrel{\circ}{W}^{1,2}\left(\Omega_{2}\right)$ and satisfies

$$
\left.(v, w)=-\lambda\left\langle\eta_{\varepsilon} \varphi, w\right\rangle+\int_{\Omega_{2}}\left(A_{i j} \partial_{x_{j}} \varphi w \partial_{x_{i}} \eta_{\varepsilon}\right) d x-\int_{\Omega_{2}} \varphi A_{i j} \partial_{x_{j}} \eta_{\varepsilon} \partial_{x_{i}} w\right) d x
$$

Choosing $w=v$ and using Hölder's inequality together with Lemma 2(i), we obtain

$$
\|\nabla v\|_{L^{2}\left(\Omega_{2}\right)} \leq C\|\nabla \varphi\|_{L^{2}\left(\Omega_{1} \backslash \Omega_{q \varepsilon}\right.}
$$

Taking into account (122), we get

$$
\|\Psi\|_{L^{2}\left(\Omega_{2}\right)} \leq C\|\nabla \varphi\|_{L^{2}\left(\Omega_{1} \backslash \Omega_{q \varepsilon}\right.}
$$

Using (121), (123) and Corollary [3, we arrive at

Corollary 6. Under the assumptions on $\Omega_{1}$ and $\Omega_{2}$ of this section, the following estimate for the eigenvalues of the problems (4) and (5) holds

$$
\left|\lambda_{m}^{-1}-\mu_{k}^{-1}\right| \leq C \max _{\varphi \in X_{m},\|\varphi\|=1}\|\nabla \varphi\|_{L^{2}\left(\Omega_{1} \backslash \Omega_{q \varepsilon}\right)}^{2}
$$

where $\mu_{1}, \ldots, \mu_{J_{m}}$ are eigenvalues of (5) located near $\lambda_{m}^{-1}$, see Proposition 3 . 


\subsection{The case of Lipschitz domains}

We recall the domain $\Omega$ is called Lipschits with Lipschitz constant less than or equal to $C_{*}$ if the boundary $\partial \Omega$ can be covered by a finite number of balls $B$ such that in an appropriate orthogonal system of coordinate $B \cap \Omega=$ $B \cap\left\{y=\left(y^{\prime}, y_{n}\right): y_{n}>h\left(y^{\prime}\right)\right\}$, where $h$ satisfies $\left|h\left(y^{\prime}\right)-h\left(z^{\prime}\right)\right| \leq C_{*}\left|y^{\prime}-z^{\prime}\right|$ and $h(0)=0$ and $B$ has the center at the origin.

We assume in this section that $\Omega_{1}$ is a bounded Lipschitz domain and we denote by $B_{k}, k=1, \ldots, M$, the balls from the covering of the boundary and by $h_{k}$ corresponding Lipschitz functions. Then there exists a positive $\delta$ such that the set $\mathcal{V}_{\delta}=\left\{x \in \mathbb{R}^{n}: \operatorname{dist}(x, \partial \Omega) \leq \delta\right\}$ contains in $\bigcup_{k=1}^{M} B_{k}$.

Concerning the domain $\Omega_{2}$ we assume that

$$
\Omega_{\varepsilon} \subset \Omega_{2} \subset \Omega_{\varepsilon}^{+}
$$

where $\varepsilon$ is a sufficiently small positive number. Moreover, we suppose that the domain $\Omega_{2}$ is Lipschitz and

$$
B_{k} \cap \Omega_{2}=B_{k} \cap\left\{y=\left(y^{\prime}, y_{n}\right): y_{n}>g_{k}\left(y^{\prime}\right)\right\} \text { for } k=1, \ldots, M \text {, }
$$

where $g_{k}$ are Lipschitz functions with the Lipschits constant $\leq C_{*}$.

Proposition 6. The following inequality

$$
\left\|T_{0} \varphi\right\|^{2} \leq C \int_{\Omega_{1} \backslash \Omega_{2}}|\nabla \varphi|^{2} d x
$$

holds for all $\varphi \in X_{m}$. Here $T_{0}=I-S_{0}$ and $S_{0}$ is the orthogonal projector onto $W^{1,2}\left(\Omega_{1} \cap \Omega_{2}\right)$, compare with Corollary 3 .

Proof. Since $\varphi \in \stackrel{\circ}{W}^{1,2}\left(\mathbb{R}^{n} \backslash\left(\Omega_{1} \cap \Omega_{2}\right)\right)$ the trace of this function on $\partial\left(\Omega_{1} \cap \Omega_{2}\right)$ belongs to $W^{1 / 1,2}\left(\partial\left(\Omega_{1} \cap \Omega_{2}\right)\right)$ and

$$
\|\varphi\|_{W^{1 / 1,2}\left(\partial\left(\Omega_{1} \cap \Omega_{2}\right)\right)} \leq C\|\varphi\|_{W^{1,2}\left(\Omega_{1} \backslash \Omega_{2}\right)} .
$$

Since $T_{0} \varphi$ is harmonic in $\Omega_{1} \cap \Omega_{2}$ with the Dirihlet boundary condition $u=\varphi$ on $\partial \Omega_{2}$ we have that

$$
\|\nabla T \varphi\|_{L^{2}\left(\Omega_{2}\right)} \leq c\|\varphi\|_{W^{1 / 1,2}\left(\partial \Omega_{2}\right)} \leq C_{1}\|\nabla \varphi\|_{L^{2}\left(\Omega_{1} \backslash \Omega_{2}\right)} .
$$

This estimate together with $T \varphi=\varphi$ outside $\Omega_{2}$ implies (124). 
The estimate obtained in the last proposition together with Corollary 2 and $T_{0} \varphi=\varphi$ outside $\Omega_{1} \cap \Omega_{2}$, implies the following

Corollary 7. Let $\Omega_{2} \subset \Omega_{1}$. Then for $j=1, \ldots, J_{m}$,

$$
c \min \|\nabla \varphi\|_{L^{2}\left(\Omega_{1} \backslash \Omega_{2}\right)}^{2} \leq\left|\mu_{m_{j}}^{-1}-\lambda_{m}^{-1}\right| \leq C \max \|\nabla \varphi\|_{L^{2}\left(\Omega_{1} \backslash \Omega_{2}\right)}^{2},
$$

where minimum and maximum are taken over all $\varphi \in X_{m}$ with $\|\varphi\|=1$.

Let us turn to estimating of the function $\Psi=\Psi_{\varphi}$. We have the following

Proposition 7. There exists a domain $S$ such that $\Omega_{2} \backslash \Omega_{1} \subset S \subset \Omega_{2}$, $|S| \leq C\left|\left(\Omega_{2} \backslash \Omega_{1}\right)\right|$ and the following inequality

$$
\left\|\nabla \Psi_{\varphi}\right\|_{L^{2}\left(\Omega_{2}\right)}^{2} \leq C_{1} \max \int_{S}|\nabla \varphi|^{2} d x
$$

holds, where $\max$ is taken over all $\varphi \in X_{m}$ satisfying $\|\varphi\|=1$. Here by $|S|$ is denote the area of $S$ and the constant $C$ is independent of $\varepsilon$.

Proof. There exists a set of smooth functions $\psi_{k} \in C^{1}\left(B_{k}\right)$ with compact support such that $\psi_{1}(x)+\cdots+\psi_{N}(x)=1$ on $\mathcal{V}_{\delta}$.

We take a smooth function $\eta=\eta(t)$ which is equal to 1 for $t<3 / 2$ and to 0 for $t>2$ and introduce the function $\zeta_{k}(x)$ in $B_{k}$ by

$$
\zeta_{k}(x)=\eta\left(\frac{y_{n}-g_{k}\left(y^{\prime}\right)}{h_{k}\left(y^{\prime}\right)-g_{k}\left(y^{\prime}\right)}\right)
$$

if $h_{k}\left(y^{\prime}\right)>g_{k}\left(y^{\prime}\right)$ and $\zeta_{k}(x)=0$ otherwise. Here the local variable $y$ is considered as a function of $x$. We define also

$$
\zeta(x)=\sum_{k=1}^{N} \zeta_{k}(x) \psi_{k}(x) .
$$

One can verify that $\zeta(x)=1$ if $x \in \Omega_{2}$ and $y_{n}-g_{k}\left(y^{\prime}\right)<3\left(h_{k}\left(y^{\prime}\right)-g_{k}\left(y^{\prime}\right)\right) / 2$. Moreover, the following inequality holds:

$$
\int|(\nabla \zeta) u(x)|^{2} d x \leq C \int_{\operatorname{supp} \zeta}|\nabla u|^{2} d x
$$


for $u \in \stackrel{\circ}{W}^{1,2}\left(\Omega_{2}\right)$. It is sufficient to prove a local version of this inequality, i.e.

$$
\int_{B_{k}}\left|\nabla_{y} \zeta_{k} u\right|^{2} d y \leq c \int_{B_{k}, 0<y_{n}-g_{k}\left(y^{\prime}\right)<2\left|h_{k}\left(y^{\prime}\right)-g_{k}\left(y^{\prime}\right)\right|}|\nabla u|^{2} d y
$$

for smooth functions $u$ equals zero for $y_{n}<g_{k}\left(y^{\prime}\right)$. Estimate (127) follows from one dimensional Hardy inequality.

Now, we are looking for the function $\Psi$ in the form $\Psi=\zeta \varphi+v$. Then $v$ satisfies the equation

$$
(v, w)=-\left\langle\varphi A_{i j} \partial_{x_{j}} \zeta, \partial_{x_{i}} w\right\rangle+\left\langle A_{i j} \partial_{x_{j}} \zeta \partial_{x_{i}} \varphi, w\right\rangle-\lambda_{m}\langle\zeta \varphi, w\rangle .
$$

Putting here $w=v$ and using (126), we get

$$
(v, v) \leq C \int_{\operatorname{supp} \zeta}|\nabla \varphi|^{2} d x
$$

which implies (125).

From Propositions [6, 7 and Corollary [3] it follows

Corollary 8. There exists a domain $S$ such that $\Omega_{2} \backslash \Omega_{1} \subset S \subset \Omega_{2},|S| \leq$ $C\left|\Omega_{2} \backslash \Omega_{1}\right|$ and the following inequality

$$
\left|\mu_{k}^{-1}-\lambda_{m}^{-1}\right| \leq C_{1} \max \int_{\left(\Omega_{1} \backslash \Omega_{2}\right) \cup S}|\nabla \varphi|^{2} d x
$$

holds, where $\max$ is taken over all $\varphi \in X_{m}$ satisfying $\|\varphi\|=1$.

\section{References}

[1] Bucur, Dorin; Buttazzo, Giuseppe, Variational methods in shape optimization problems. Progress in Nonlinear Differential Equations and their Applications, 65. Birkhäuser Boston, Inc., Boston, MA, 2005.

[2] Burenkov, Victor I.; Lamberti, Pier Domenico Spectral stability of Dirichlet second order uniformly elliptic operators. J. Differential Equations 244 (2008), no. 7, 1712-1740.

[3] Delfour, M. C.; Zolésio, J.-P. Shapes and geometries. Analysis, differential calculus, and optimization. Advances in Design and Control, 4. Society for Industrial and Applied Mathematics (SIAM), Philadelphia, PA, 2001. 
[4] P.R. Garabedian, M. Schiffer, Convexity of domain functionals, J. Anal. Math. 2 (1953), 281368.

[5] P. Grinfeld, G.Strang, Laplace eigenvalues on polygons, Comput. Math. Appl. 48 (2004), 1121-1133.

[6] Grinfeld, P. Hadamard's formula inside and out. J. Optim. Theory Appl. 146 (2010), no. 3, 654690.

[7] Grinfeld, Pavel; Strang, Gilbert Laplace eigenvalues on regular polygons: a series in 1/N. J. Math. Anal. Appl. 385 (2012), no. 1, 135149,

[8] Hadamard, J.,Mémoire sur le problème d'analyse relatif à l'équilibre des plaques élastiques encastrées, Mémoires des Savants Entrangers 33 (1908).

[9] Hadamard, J., Mémoire sur le problème d'analyse relatif à l'équilibre des plaques élastiques encastrées, (Euvres, 2 (1968), 515-631.

[10] Henrot, Antoine, Extremum problems for eigenvalues of elliptic operators. Frontiers in Mathematics. Birkhäuser Verlag, Basel, 2006.

[11] Kenig, Carlos E., Harmonic analysis techniques for second order elliptic boundary value problems. CBMS-AMS Regional Conference Series in Mathematics, No. 83, 1994.

[12] Kozlov, Vladimir, On the Hadamard formula for nonsmooth domains. J. Differential Equations 230 (2006), no. 2, 532-555.

[13] Kozlov, Vladimir, $L^{q}$-perturbations of leading coefficients of elliptic operators: asymptotics of eigenvalues. Abstr. Appl. Anal. 2006, Art. ID $26845,15 \mathrm{pp}$.

[14] Kozlov, V. and Nazarov, S., On the Hadamard formula for second order systems in non-smooth domains, to appear in CPDE.

[15] Kawohl, B., Some nonconvex shape optimization problems. Optimal shape design (Tria, 1998), 7-46, Lecture Notes in Math., 1740, Springer, Berlin, 2000.

[16] J.W.S.Rayleigh, The Theory of Sound vol. I, Macmillan and Co, London, 1894. 
[17] M. van den Berg, On Rayleigh's formula for the first Dirichlet eigenvalue of a radial perturbation of a ball. To appear in Journal of Geometric Analysis. 